\title{
Influencia de medios de cultivo y niveles de pH en el crecimiento in vitro de 6 cepas de 3 especies del género Suillus.
}

\author{
Patricio Chung G. ${ }^{1 \text { * }}$ \\ ${ }^{1}$ Instituto Forestal, Sede Biobío. pchung@infor.cl. \\ *Autor de correspondencia \\ DOI: https://doi.org/10.52904/0718-4646.2021.555
}

Recibido: 09.12.2021; Aceptado 22.12.2021

\begin{abstract}
RESUMEN
Se analizó el comportamiento in vitro de 6 cepas de las especies Suillus luteus, S, granulatus y S. bellinii, asociados a plantaciones de Pinus radiata. Se evaluó las variables crecimiento radial (CR), velocidad media de crecimiento(VMC) y biomasa (B) creciendo en los medios PDA (Papa Dextrosa Agar), MNM (Medio Melin-Norkrans) y BAF (Biotina Aneurina ácido Fólico) y en 5 niveles de $\mathrm{pH}(4,8,5,3 ; 5,8 ; 6,3 ;$ y 6,8$)$. A nivel de cepas se analizó las interacciones presentes entre los factores medio de cultivo y $\mathrm{pH}$. Se observó comportamientos variables de cada cepa para las variables CR y VMC frente a los factores evaluados. Para la variable $B$, se observan promedios significativos para los medios de cultivo PDA y/o BAF, dependiendo de la cepa estudiada, con valores significativos de pH que variaron para cada cepa. Comparaciones entre valores del CR y de B, muestran alta correlación entre ellos para cepas de S. granulatus, baja para cepas de $S$. luteus y mediana a nula para cepas de $S$. bellinii.
\end{abstract}

Palabras claves: Suillus, cepas, pruebas de crecimiento, in vitro

\section{SUMMARY}

The in vitro behavior of 6 strains of the species Suillus luteus, S. granulatus and S. bellinii, associated with Pinus radiata plantations, was analyzed. The variables radial growth (CR), average growth speed (VMC) and biomass (B) growing in PDA (Potato Dextrose Agar), MNM (Melin-Norkrans Medium) and BAF (Biotin Aneurin Folic acid) media and in $5 \mathrm{pH}$ levels $(4.8,5.3,5.8,6.3$, and 6.8) were evaluated. At the strain level, the interactions between the culture medium and $\mathrm{pH}$ levels were analyzed. Variable behaviors of each strain were observed for the $\mathrm{CR}$ and VMC variables. For variable $\mathrm{B}$, statiscally significant differences were observed for the PDA and / or BAF culture media, depending on the strain studied, with significant $\mathrm{pH}$ values that varied for each strain. Comparisons between $\mathrm{CR}$ and $\mathrm{B}$ values show high correlation between them for $S$. granulatus strains, low for $S$. luteus strains and medium to null for $S$. bellinii strains.

Keywords: Suillus, strains, growth testing, in vitro

\section{INTRODUCCIÓN}

El uso de los recursos micológicos del bosque comenzó con los pueblos originarios que los utilizaban para alimentación. En la actualidad este uso de ha masificado, llegando a constituir los hongos silvestres comestibles un recurso natural de gran importancia económica y social.

La riqueza micológica nativa se vio aumentada con la introducción de especie forestales como Pinus radiata, en cuyas plantaciones se desarrolla una variedad de especies fúngicas, dentro de las cuales destacan Lactarius deliciosus y especies del género Suillus. Estas especies fúngicas acompañantes del pino han sido de gran importancia en el contexto social y económico de muchas familias rurales, y en la actualidad representan para el país exportaciones comerciales con valores cercanos a los US $\$ 3,8$ MM para Suillus spp. y US\$137 mil para Lactarius deliciosus (INFOR, 2020).

Los hongos del género Suillus conviven simbióticamente con las raíces de árboles de Pinus radiata, principal especie plantada en Chile. Sin embargo, para asegurar su presencia resultan importantes los trabajos de micorrización, especialmente en la etapa de viverización de las plantas. 
En la actualidad, los hongos comestibles del género Suillus, y en especial las especies de mayor presencia en plantaciones de pino como son S. luteus, S. granulatus y S. bellinii, constituyen un recurso valioso, cuyo uso como alimento o insumo comercial ha adquirido gran importancia en las zonas rurales, gracias a la necesidad de cubrir el aumento en la demanda de productos de tipo orgánico y de tipo funcional. Su recolección es desarrollada por miles de personas y/o familias que ven en esta actividad una forma de mejorar su calidad de vida, siendo, el eslabón principal de la cadena de comercialización

Las especies del género Suillus son hongos simbiontes catalogado dentro del grupo de los hongos pioneros (Bruns et al., 2002; Blanco et al., 2012) del tipo ectomicorrícico y que desarrollan su ciclo de vida en bosques de pino presentes en buena parte de nuestro territorio nacional. La proliferación de sus cuerpos fructíferos ocurre principalmente en los meses de otoño, pudiendo aparecer en menor medida en la época de primavera, cuando las condiciones ambientales permiten su desarrollo. Además de su importancia económica como PFNM, poseen como todo hongo micorrícico una importancia ecológica dentro de los ecosistemas forestales, por lo que cualquier actividad dentro del bosque o eventos que sean causados en forma natural o antrópica que alteren el suelo tendrían un importante impacto en la supervivencia y fructificación de estos hongos.

La relación simbiótica entre hongos y especies forestales constituye una ventajosa oportunidad para implementar líneas de investigación y desarrollo innovativos, que conjuguen la recuperación de suelos degradados con la restauración y enriquecimiento del bosque, mejorando el desempeño de las plantaciones, con la generación de productos intermedios de alto valor económico, ecológico y social, como son los hongos ectomicorrícicos comestibles. Sin embargo, la producción natural de estos hongos en el bosque es variable, de modo que el interés por obtener una producción alta y estable, ha motivado iniciativas para cultivarlos mediante el establecimiento de plantas inoculadas con cepas adaptadas a condiciones medioambientales específicas (Chung, 2020).

En relación a lo anterior, y para llegar a determinar las condicionas ideales para que se establezca y desarrolle el hongo en conjunto con la planta, se ha hecho necesario una serie de investigaciones en torno al desarrollo de una planta ideal inoculada con el hongo, que pueda desarrollar esta simbiosis conjuntamente con el desarrollo de hongos comestibles, previo y posterior a las labores de plantación.

Para llevar a cabo los trabajos de inoculación de plantas con hongos ectomicorrícicos específicos, se deben realizar un conjunto de investigaciones para desarrollar protocolos que posibiliten el contacto y una exitosa unión hongo-planta dando lugar a las formaciones ectomicorrícicas. Para ello, uno de los aspectos importantes, es la elaboración de material inoculante y dentro de este, la cepa de hongo previamente seleccionada y masificada bajo parámetros ambientales y químicos fijadas en laboratorio, como son el $\mathrm{pH}$ y la disponibilidad de nutrientes.

Para tales efectos se requiere disponer de un banco de cultivos puros con cepas nacionales, donde abastecerse y seleccionar material de acuerdo a características específicas de uso. Por este motivo, el Instituto Forestal ha establecido un banco de cepas, con el propósito de generar una masa crítica para la elaboración de productos que permitan generar diversos formatos de material inoculante fúngico, que puedan ser utilizados en la producción de plantas y posibilitar así mejorar la rentabilidad de plantaciones forestales a través de una producción de hongos ectomicorrícicos comestibles de alto valor económico y social.

Por lo tanto, para los trabajos de inoculación con hongos micorrícicos, se requiere de inoculantes fúngicos a gran escala, por estas razones es necesario definir la composición óptima del medio de cultivo para cada hongo tomando en cuenta las diferentes cepas una gran variación de condiciones del suelo (Islam \& Ohga, 2013).

Consecuentemente, el presente trabajo busca investigar el desempeño de 3 especies del género Suillus (S. luteus, S. granulatus y $S$. bellinii) y sus respectivas cepas (2 cepas por especie) bajo diferentes condiciones controladas de cultivo en condiciones in vitro, evaluando el efecto de 3 medios de cultivo (PDA, MMN y BAF) y 5 niveles de $\mathrm{pH}(4,8 ; 5,3 ; 5,8 ; 6,3$ y 6,8$)$ sobre el crecimiento radial (CR), velocidad media de crecimiento (VMC) y peso seco de la biomasa (B) producida por cada una de las especies y cepas. 


\section{MATERIALES Y METODOS}

Se utilizaron 2 cepas de cada uno de los hongos comestibles ectomicorrícicos Suillus luteus L. Gray, $S$. granulatus (L.) Roussel y S. bellinii (Inz.) Kuntze (Figura 1), procedentes del banco de cepas del Instituto Forestal de Chile (INFOR) y cuyo material fue colectado desde plantaciones de Pinus radiata en diferentes regiones de nuestro país (Cuadro 1).

Los cultivos puros fueron obtenidos de aislados usando la técnica descrita por Molina y Palmer (1982), generados a partir del sombrero de un esporocarpo joven y cultivados sobre medio MMN en agar (MelinNorkrans modificado), bajo oscuridad y a temperatura de $23^{\circ} \mathrm{C}$.

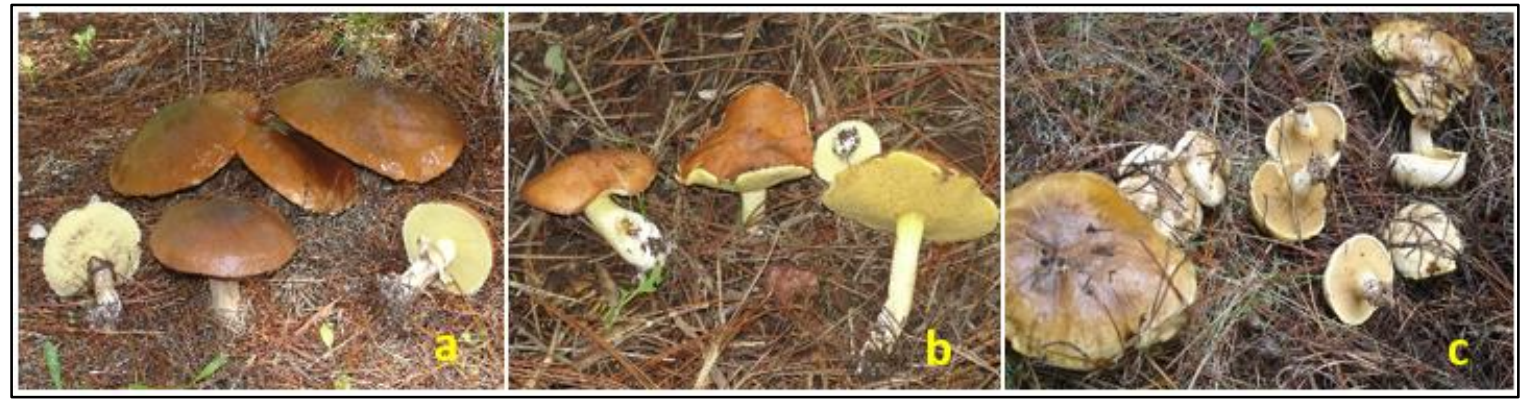

Figura 1. Imágenes de Suillus luteus (a), S. granulatus (b) y S. bellinii (c) en su ambiente bajo plantaciones de pino

En una etapa preliminar, el material original de cada cepa fue activado y masificado en discos de Petri con medio Extracto de Malta- Agar (EMA) al 2\%. Para ello, se extrajo desde el material original discos de $5 \mathrm{~mm}$, los que fueron puestos en el centro de placas petri con $20 \mathrm{ml}$ del medio nutritivo durante 30 días, período en el cual se generó suficiente tejido miceliar para ser utilizados en la instalación del estudio respectivo.

Para la instalación del estudio, los medios de cultivos usados fueron: (i) PDA, extracto de papa dextrosa agar (Difco, Bencton Dickinson and Company, USA); (ii) MNM, Melin- Norkrans (Marx, 1969); y (iii) BAF, Biotina Aneurina Ácido Fólico (Moser, 1960). Sobre ellos se evaluó el comportamiento de cada cepa en relación a su crecimiento radial $(\mathrm{CR})$ en milímetros, velocidad media de crecimiento (VMC) en milímetros por día y biomasa (B) en miligramos y en 5 niveles de $\mathrm{pH}(4,8 ; 5,3 ; 5,8 ; 6,3$ y 6,8$)$, usando un diseño estadístico completamente aleatorizado con cinco repeticiones de cada tratamiento.

Cuadro 1. Cepas y antecedentes de las especies utilizadas (S. luteus, S. granulatus y S. bellinii)

\begin{tabular}{|c|c|c|c|c|c|c|c|}
\hline \multirow[b]{2}{*}{ Especie } & \multirow[b]{2}{*}{$\begin{array}{l}\text { Código } \\
\text { de cepa }\end{array}$} & \multirow{2}{*}{$\begin{array}{l}\text { Región, } \\
\text { Provincia, } \\
\text { Comuna }\end{array}$} & \multicolumn{2}{|c|}{ Ubicación } & \multirow{2}{*}{$\begin{array}{l}\text { Tipo de } \\
\text { Suelo }\end{array}$} & \multirow[b]{2}{*}{ Hospedante } & \multirow[b]{2}{*}{$\begin{array}{l}\text { Edad } \\
\text { rodal } \\
\text { (años) }\end{array}$} \\
\hline & & & $\begin{array}{l}\text { Lat }\left({ }^{\circ} \mathrm{S}\right) \text {, } \\
\text { Long }\left({ }^{\circ} \mathrm{O}\right)\end{array}$ & $\begin{array}{c}\text { Altitud } \\
\text { (msnm) }\end{array}$ & & & \\
\hline \multirow[b]{2}{*}{$\begin{array}{l}\text { Suillus } \\
\text { luteus }\end{array}$} & IF1618002 & $\begin{array}{l}\text { Ñuble, Itata, } \\
\text { Coelemu }\end{array}$ & $\begin{array}{l}36^{\circ} 28^{\prime} 41,90^{\prime \prime} \mathrm{S} \\
72^{\circ} 42^{\prime} 15,34^{\prime \prime} \mathrm{O}\end{array}$ & 29 & $\begin{array}{l}\text { Franco } \\
\text { arenoso }\end{array}$ & P. radiata & 8 \\
\hline & IF732003 & $\begin{array}{l}\text { Maule, Talca, } \\
\text { Constitución }\end{array}$ & $\begin{array}{l}35^{\circ} 34^{\prime} 20,94^{\prime \prime} \mathrm{S} \\
72^{\circ} 36^{\prime} 08,20^{\prime \prime} \mathrm{O}\end{array}$ & 25 & $\begin{array}{l}\text { Franco } \\
\text { arcillo } \\
\text { arenoso }\end{array}$ & P. radiata & 5 \\
\hline \multirow{2}{*}{$\begin{array}{l}\text { Suillus } \\
\text { granulatus }\end{array}$} & IF937002 & $\begin{array}{l}\text { Araucanía, } \\
\text { Cautín, Nueva } \\
\text { Imperial }\end{array}$ & $\begin{array}{l}38^{\circ} 42^{\prime} 52,23^{\prime \prime} \mathrm{S} \\
73^{\circ} 01^{\prime} 34,57^{\prime \prime} \mathrm{O}\end{array}$ & 65 & $\begin{array}{l}\text { Limo } \\
\text { arcilloso }\end{array}$ & P. radiata & 8 \\
\hline & IF1616002 & $\begin{array}{l}\text { Nuuble, Diguillín, } \\
\text { Quillón }\end{array}$ & $\begin{array}{l}36^{\circ} 42^{\prime} 10,86^{\prime \prime} \mathrm{S} \\
72^{\circ} 30^{\prime} 27,43^{\prime \prime} \mathrm{O}\end{array}$ & 536 & $\begin{array}{l}\text { Franco } \\
\text { arenoso }\end{array}$ & P. radiata & 10 \\
\hline \multirow{2}{*}{$\begin{array}{l}\text { Suillus } \\
\text { bellinii }\end{array}$} & IF633001 & $\begin{array}{l}\text { O'Higgins, } \\
\text { Cardenal Caro, } \\
\text { Marchihue }\end{array}$ & $\begin{array}{l}34^{\circ} 21^{\prime} 29,70^{\prime \prime} \mathrm{S} \\
71^{\circ} 53^{\prime} 46,70^{\prime \prime} \mathrm{O}\end{array}$ & 440 & $\begin{array}{l}\text { Franco } \\
\text { arcilloso }\end{array}$ & P. radiata & 15 \\
\hline & IF713001 & $\begin{array}{l}\text { Maule, Linares, } \\
\text { Longaví }\end{array}$ & $\begin{array}{l}36^{\circ} 10^{\prime} 58,02^{\prime \prime} \mathrm{S} \\
71^{\circ} 32^{\prime} 12,89^{\prime \prime} \mathrm{O}\end{array}$ & 332 & $\begin{array}{l}\text { Franco } \\
\text { limoso }\end{array}$ & P. radiata & 4 \\
\hline
\end{tabular}

Los medios de cultivo utilizados se esterilizaron en autoclave a $121^{\circ} \mathrm{C}$, a 1,2 atm de presión, por 30 minutos, ajustando previamente sus valores respectivos de $\mathrm{pH}$ con $\mathrm{HCL} \circ \mathrm{KOH} 1 \mathrm{~N}$ y con mediciones 
realizadas con un peachímetro marca Thermo Scientific Orion modelo Star A111. Finalizado el proceso de esterilización, estos fueron llevados a una Cámara de Flujo Laminar marca Filtromet modelo H24302, de fabricación nacional, donde se realizó el vaciado de los medios de cultivo a discos de Petri de $90 \times 15$ $\mathrm{mm}$, ayudados por una jeringa de $25 \mathrm{ml}$, vertiendo $20 \mathrm{ml}$ de medio en cada disco. Posteriormente, se dejó enfriar en ambiente estéril hasta su uso.

El proceso de instalación de los ensayos se realizó con la ayuda de un sacabocado que permitió obtener segmentos circulares de $5 \mathrm{~mm}$ desde las placas que contenían el material miceliar madre. Estos segmentos fueron dispuestos en el centro de los discos de Petri correspondientes a cada tratamiento, procediendo luego a sellarlos con cintas de parafilm y marcarlos con el nombre de la especie, código de cepa, número de repetición, medio de cultivo, nivel de $\mathrm{pH}$ y fecha de instalación.

Una vez finalizada la operación de instalación de los ensayos en los discos con los segmentos miceliares respectivos, estos se ingresaron a una Cámara de Crecimiento marca Forma Scientific modelo 3744, en oscuridad y a $24^{\circ} \mathrm{C}$ de temperatura por 30 días.

Para realizar las mediciones de crecimiento radial (CR) se utilizó un pie de metro digital marca Ubermann. Cada medición radial se realizó en 4 direcciones a partir del centro donde se ubicó el disco de micelio, registrándose el crecimiento de las cepas en cada uno de los discos de cada tratamiento cada 3 días por un lapso de 30 días.

Para obtener los valores de biomasa seca (B) al final del período de evaluación, se procedió a extraer desde los discos de Petri, el micelio obtenido junto con el medio con agar.

Para eliminar el agar, se aplicó la metodología utilizada por Santiago-Martínez et al. (2003), que consistió en eliminar el agar por calentamiento en baño maría y luego enjuagando la colonia con agua caliente, para después secarla en estufa a $60^{\circ} \mathrm{C}$ por 48 horas hasta peso constante.

Posteriormente se pesó cada muestra, descontando el peso del papel y del inóculo inicial, obteniendo así la biomasa seca producida en cada tratamiento (Figura 2).

Para determinar la velocidad media de crecimiento (VMC) de las cepas, se usó los datos obtenidos de mediciones de crecimiento radial cada 3 días por 30 días, los que se ajustaron mediante una ecuación de regresión lineal para calcular la pendiente de la curva de crecimiento y obtener el promedio de crecimiento del hongo por día (Santiago- Martínez et al., 1995).

El análisis de los datos se realizó mediante análisis de varianza (ANDEVA), utilizando para ello, el software estadístico INFOSTAT versión 2015p.

La homogeneidad de varianza se evaluó mediante la prueba de Levene $(P \leq 0,05)$. En tanto que el supuesto de normalidad de los residuos se evaluó a través de la prueba de Shapiro-Wilks $(P \leq 0,05)$.

Por otro lado, para detectar diferencias significativas entre los tratamientos, se aplicó la prueba de comparaciones múltiples de Tukey (Montgomery,1984), con $\mathrm{P} \leq 0,05$.

Para visualizar la relación entre las variables analizadas, se calculó el coeficiente de correlación de Pearson que indica el grado de linealidad existente entre pares de variables. 


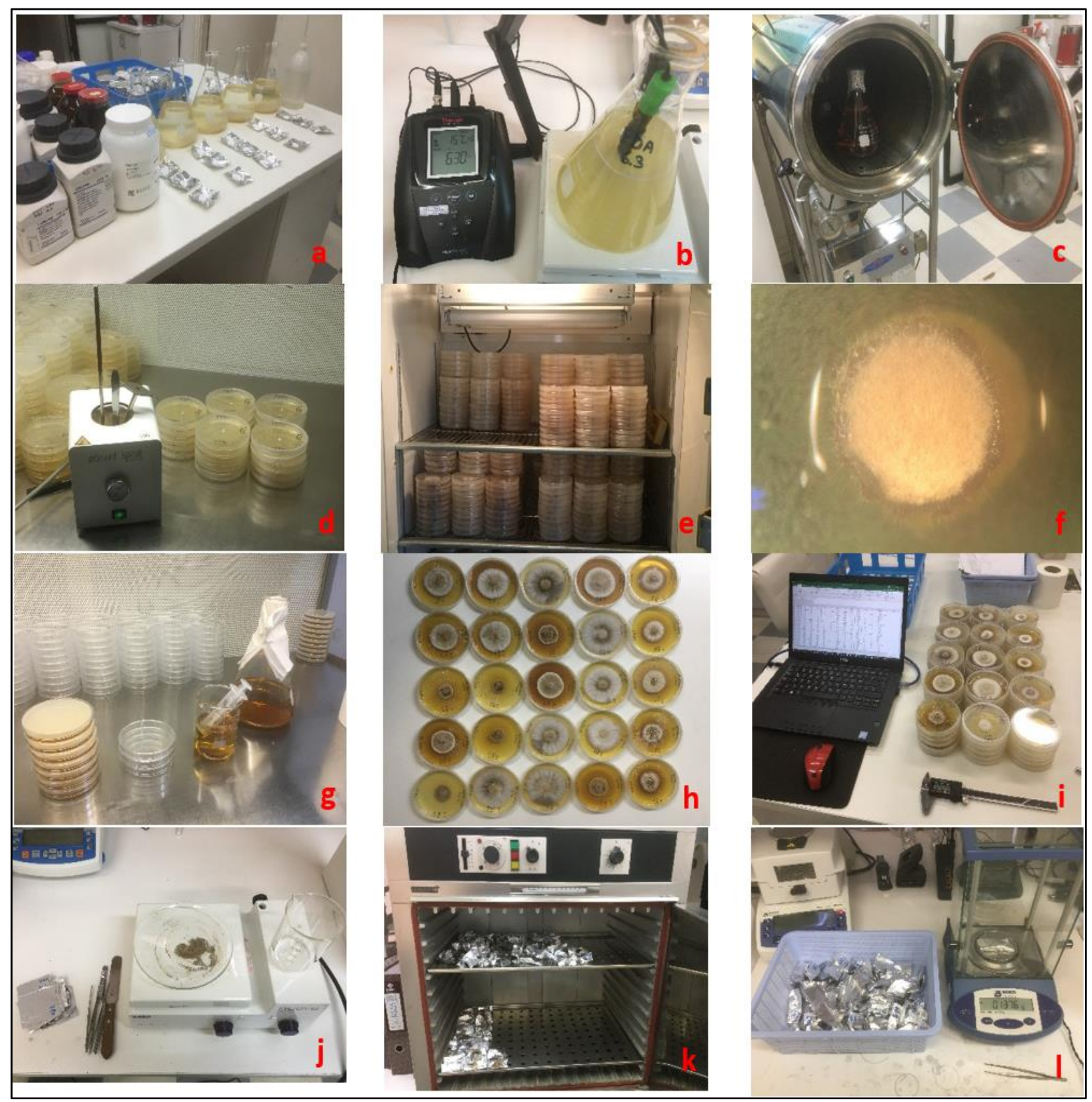

Figura 2. Preparación de los medios de cultivo (a); Ajuste de pH de los medios de cultivo (b); Esterilización de medios de cultivo en autoclave (c); Estableciendo los tratamiento con la ayuda de un sacabocado (d); Discos de petri con los diferentes tratamientos en cámara de crecimiento a $24^{\circ} \mathrm{C}$ (e); Segmento circular de $5 \mathrm{~mm}$ extraído con sacabocado y utilizado como inoculante (f); Vaciado de medios a discos de Petri (g); Aspecto de crecimiento de cepa de S. granulatus a distintos PH en medio PDA (h); Medición del crecimiento radial de cada cepa y su tratamiento (i); Extracción de agar desde micelio crecido en discos de Petri (j); Colocación de micelio puro en papel de aluminio y secado a $60{ }^{\circ} \mathrm{C}$ pos 48 horas $(\mathrm{k})$; Pesaje de micelio puro en papel de aluminio previamente pesado (l).

\section{RESULTADOS}

\section{Análisis de Suillus luteus}

\section{-A nivel de cepas}

Los ANDEVA de los datos obtenidos para las cepas IF732002 y IF1618002, arrojan interacciones significativas entre los factores nivel de $\mathrm{pH}$ y medio de cultivo con efectos sobre las variables crecimiento radial $(\mathrm{CR})$ y velocidad media de crecimiento (VMC), mientras que para el peso seco de la biomasa (B), sólo se observó interacciones para la cepa IF732002 (cuadro 2). 
Cuadro 2. Significancia estadística del análisis de varianza (ANDEVA) para los valores medios obtenidos de las variables crecimiento radial (CR), velocidad media de crecimiento (VMC) y biomasa (B) para cepas de Suillus luteus.

\begin{tabular}{ccccccc}
\hline \multirow{2}{*}{ Factor } & \multicolumn{3}{c}{ Cepa IF732002 } & \multicolumn{3}{c}{ Cepa IF1618002 } \\
\cline { 2 - 7 } & $\begin{array}{c}\mathbf{C R} \\
(\mathbf{m m})\end{array}$ & $\begin{array}{c}\text { VMC } \\
(\mathbf{m m} / \mathbf{d i ́ a})\end{array}$ & $\begin{array}{c}\mathbf{B} \\
(\mathbf{m g})\end{array}$ & $\begin{array}{c}\mathbf{C R} \\
(\mathbf{m m})\end{array}$ & $\begin{array}{c}\text { VMC } \\
(\mathbf{m m} / \mathbf{d i ́ a})\end{array}$ & $\begin{array}{c}\mathbf{B} \\
(\mathbf{m g})\end{array}$ \\
\hline Medio & $<0,0001^{*}$ & $<0,0001^{*}$ & $<0,0001^{*}$ & $0,0010^{*}$ & $<0,0001^{*}$ & 0,2884 \\
\hline $\mathrm{pH}$ & $<0,0001^{*}$ & $<0,0001^{*}$ & $0,0001^{*}$ & 0,6073 & 0,6714 & 0,6571 \\
\hline Medio $\times \mathrm{pH}$ & $0,0025^{*}$ & $0.0042^{*}$ & $0,0341^{*}$ & $0,0303^{*}$ & $0,0180^{*}$ & 0,2290 \\
\hline${ }^{*}$ Efectos significativos $(P \leq 005)$ & & & & &
\end{tabular}

-Crecimiento Radial: La cepa IF732002 evidenció mayor crecimiento en el medio PDA a pH 5,8, sin diferenciarse significativamente del observado en PDA a pH 5,3 y 6,3 y en $\mathrm{MMN}$ a pH 6,8, observándose además una baja en el crecimiento a pH 4,8 en los tres medios utilizados.

Respecto al pH, se presentó mayor crecimiento a 5,8, con diferencias significativas sólo con valores de 5,3 y 4,8. A nivel de medios de cultivo, PDA presentó el mejor desempeño. Para la cepa IF1618002 se observó mayor crecimiento en el medio MMN a pH 6,3, en tanto el medio BAF a pH 6,8 presentó el menor crecimiento entre todos los tratamientos aplicados. Respecto al pH, este no presentó diferencias significativas entre los diferentes niveles (cuadro 3).

- Velocidad Media de Crecimiento: Para la cepa IF32002 el comportamiento fue similar al observado en el crecimiento radial; crecimiento más rápido en el medio PDA a $\mathrm{pH} 5,8$, pero sin presentar diferencias significativas a pH 5,3 y 6,3 dentro del mismo medio, y tampoco con los medios $\mathrm{MMN}$ a pH 6,8 y BAF a $\mathrm{pH}$ 5,8. La menor velocidad de crecimiento miceliar se observó a pH 4,8 diferenciándose significativamente de los demás niveles evaluados.

En medio PDA el crecimiento fue significativamente más rápido que en los restantes. En el caso de la cepa IF 1618002, no se observó diferencias significativas entre los niveles de pH dentro de cada medio de cultivo, pero sí entre ellos.

La mayor VMC se obtuvo en el medio MMN a pH 6,8, siendo el medio MMN significativamente mejor que los medios restantes independientemente del nivel de PH (cuadro 3).

-Biomasa: En la cepa IF732002, la mayor cantidad de materia seca se obtuvo en el medio BAF a pH 5,8, con rendimientos también altos para los medios PDA y BAF, en comparación al medio MMN que obtuvo los peores promedios.

No se observó diferencias entre los tratamientos que consideran al medio PDA con pH 5,3 y 6,3 y el medio BAF con pH 5,3 y 6,8, los cuales no se diferencian significativamente del tratamiento con mayor producción de biomasa seca.

La biomasa fue significativamente menor a pH 4,8. Con respecto a la cepa IF1618002, esta no presentó diferencias significativas entre los diferentes tratamientos (cuadro 3).

-Correlaciones entre variables: En ninguna de las cepas se observó una correlación elevada entre la producción de biomasa seca con el crecimiento radial ni con la velocidad media de crecimiento, observándose coeficientes de correlación que fluctúan entre 0,27 y 0,35. 
Cuadro 3. Efecto del medio de cultivo y $\mathrm{pH}$ sobre los valores medios $(\mathrm{m})$ y desviaciones estándar (DE) de las variables crecimiento radial, velocidad media de crecimiento y producción de biomasa a nivel de cepas de Suillus luteus.

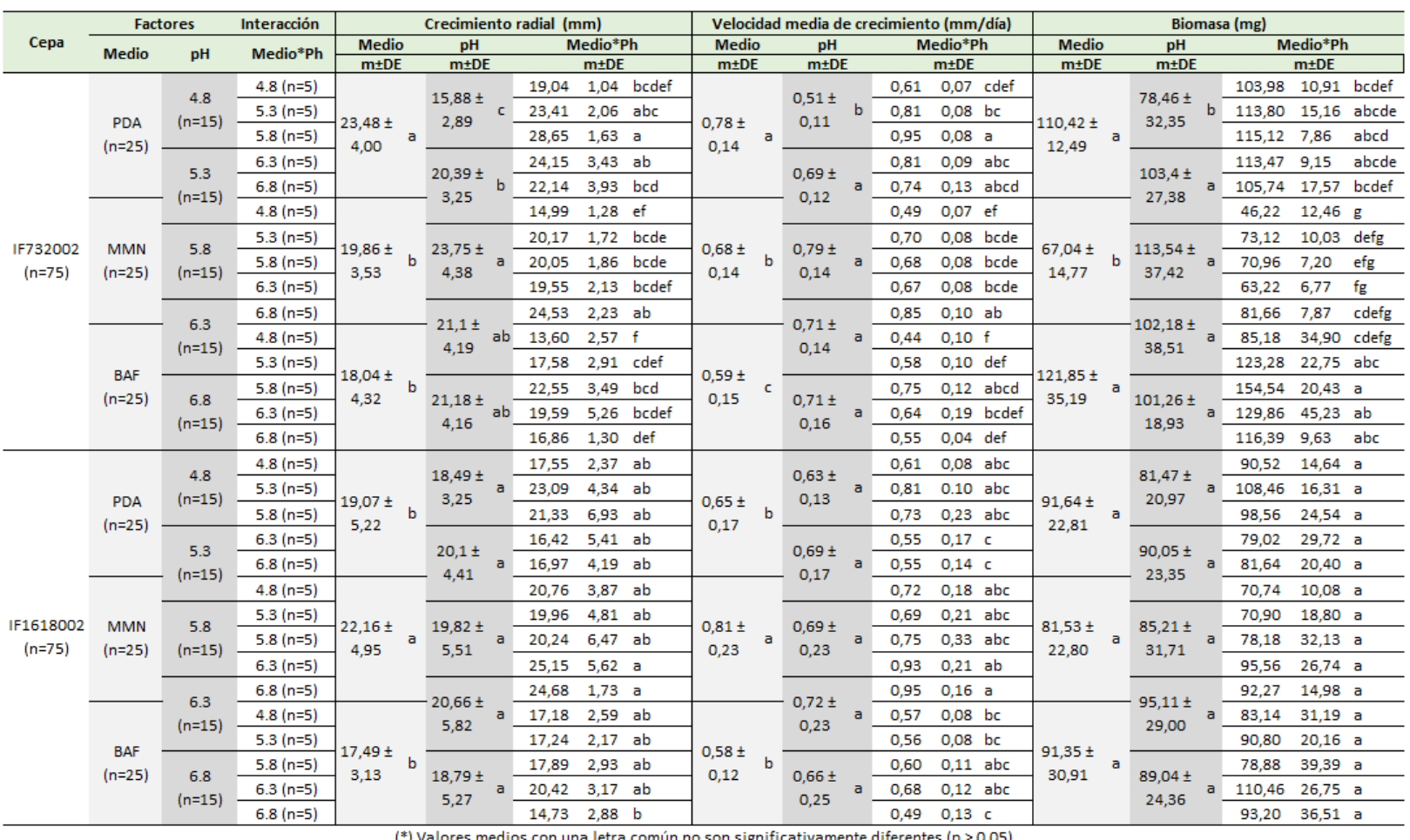

\section{-A nivel de especie}

-Crecimiento Radial: El mejor desempeño se observó en medio MMN a pH 6,8 y en PDA a 5,8. En MMN el crecimiento estuvo directamente relacionado con el nivel de $\mathrm{pH}$, mientras que en PDA y BAF, los mayores crecimientos se concentraron en los valores intermedios de $\mathrm{pH}$. El menor crecimiento se obtuvo en BAF a pH 6,8, sin diferenciarse significativamente de los valores obtenidos en los restantes niveles de $\mathrm{pH}$. Considerando sólo el $\mathrm{pH}$, el ajuste de los medios a 4,8, fue el que presentó el menor crecimiento con diferencias significativas con los demás valores de $\mathrm{pH}$, a excepción del pH 6,8 (cuadro 4).

- Velocidad Media de Crecimiento: Esta variable obtuvo su mayor valor en el medio MMN a pH 6,8, siendo el único medio que presentó diferencias al interactuar con los diferentes niveles de $\mathrm{pH}$. Los menores valores se ubicaron en los valores extremos de $\mathrm{pH}$ del medio BAF. La velocidad media de crecimiento tuvo un comportamiento muy parecido al crecimiento radial, mostrando estas dos variables un desarrollo menor en el medio BAF para los valores extremos de $\mathrm{pH}$. De lo observado a nivel de la variable $\mathrm{pH}$, se observa que el ajuste a 4,8, mostró el menor rendimiento, con diferencias significativas con los demás valores. A nivel de medios de cultivo, los medios MMN y PDA, tuvieron los mejores desempeños para esta variable (cuadro 4).

-Biomasa: La mayor biomasa seca se obtuvo en los medios BAF y PDA, los cuales no presentaron diferencias significativas para todo el rango de $\mathrm{pH}$ dentro de ellos, como tampoco entre ellos, con un máximo en el medio BAF a pH 6,3. Se agrega también el medio MMN a pH 6,8, el cual tampoco presentó diferencias con los promedios de los otros medios de cultivo (cuadro 4). A nivel de pH, el ajuste a 4,8 presentó el menor monto en materia seca, presentando diferencias significativas sólo con los ajustes a $\mathrm{pH} 5,8$ y 6,3 (cuadro 4).

Correlaciones entre Variables: Existe una correlación muy débil $(0,13)$ entre CR y B y casi inexistente $(0,02)$ entre VMC y $\mathrm{B}$. 
Cuadro 4. Efecto del medio de cultivo y $\mathrm{pH}$ sobre los valores medios $(\mathrm{m})$ y desviaciones estándar (DE) de las variables crecimiento radial, velocidad media de crecimiento y producción de biomasa para Suillus luteus.

\begin{tabular}{|c|c|c|c|c|c|c|c|c|c|c|c|c|c|c|c|c|c|c|}
\hline \multicolumn{2}{|c|}{ Factores } & \multirow{2}{*}{$\begin{array}{c}\text { Interacción } \\
\text { Medio*Ph }\end{array}$} & \multicolumn{5}{|c|}{ Crecimiento radial (mm) } & \multicolumn{6}{|c|}{ Velocidad media de crecimiento (mm/día) } & \multicolumn{5}{|c|}{ Biomasa (mg) } \\
\hline & \multirow{4}{*}{$\begin{array}{c}\mathrm{pH} \\
\begin{array}{c}4.8 \\
(n=30)\end{array}\end{array}$} & & $\begin{array}{c}\text { Medio } \\
\mathrm{m} \pm \mathrm{DE}\end{array}$ & $\begin{array}{c}\mathrm{pH} \\
\mathrm{m} \pm \mathrm{DE}\end{array}$ & & $\begin{array}{l}\text { edio }{ }^{*} p \\
m \pm D E\end{array}$ & & $\begin{array}{c}\text { Medio } \\
\mathrm{m} \pm \mathrm{DE}\end{array}$ & $\begin{array}{c}\mathrm{pH} \\
\mathrm{m} \pm \mathrm{DE}\end{array}$ & & & $\begin{array}{l}\text { ledio* } p \\
m \pm D E\end{array}$ & & $\begin{array}{c}\text { Medio } \\
\mathrm{m} \pm \mathrm{DE}\end{array}$ & $\begin{array}{c}\mathrm{pH} \\
\mathrm{m} \pm \mathrm{DE}\end{array}$ & & $\begin{array}{l}\text { ledio* } p \\
m \pm D E\end{array}$ & \\
\hline \multirow{5}{*}{$\begin{array}{c}\text { PDA } \\
(n=50)\end{array}$} & & $4.8(n=10)$ & \multirow{5}{*}{$\underset{5,11}{21,27 \pm}$ a } & \multirow{3}{*}{$\underset{3,30}{17,18 \pm} b$} & 18,29 & 1,90 & bc & \multirow{5}{*}{$\begin{array}{c}0,72 \pm \\
0,17\end{array}$} & \multirow{3}{*}{\multicolumn{2}{|c|}{$\begin{array}{c}0,57 \pm \\
0,13\end{array}$}} & 0,61 & 0,07 & bcde & \multirow{5}{*}{$\begin{array}{c}101,03 \pm \\
20,51\end{array}$} & \multirow{3}{*}{$\begin{array}{c}79,96 \pm \\
26,83\end{array}$} & 97,25 & 14,09 & $a b c d$ \\
\hline & & $5.3(n=10)$ & & & 23,25 & 3,21 & $a b$ & & & & 0,81 & 0,08 & $a b c$ & & & 111,13 & 15,11 & $a b c$ \\
\hline & & $5.8(n=10)$ & & & 24,99 & 6,12 & a & & & & 0,84 & 0,20 & $\mathrm{ab}$ & & & 106,84 & 19,27 & $a b c$ \\
\hline & \multirow{3}{*}{$\begin{array}{c}5.3 \\
(n=30)\end{array}$} & $6.3(n=10)$ & & \multirow{3}{*}{$\begin{array}{c}20,24 \pm \\
-3,81\end{array}$} & 20,28 & 5,90 & $a b c$ & & $0.69 \pm$ & & 0,68 & 0,19 & abcde & & & 96,25 & 27,56 & abcd \\
\hline & & $6.8(n=10)$ & & & 19,55 & 4,70 & $a b c$ & & $0,09 \pm$ & a & 0,65 & 0,16 & bcde & & 25,91 ab & 93,69 & 21,99 & $a b c d$ \\
\hline \multirow{5}{*}{$\begin{array}{l}\text { MMN } \\
(n=50)\end{array}$} & & $4.8(n=10)$ & \multirow{5}{*}{$\underset{4,41}{21,01 \pm} a$} & & 17,87 & 4,08 & $b c$ & \multirow{5}{*}{$\begin{array}{c}0,74 \pm \\
0,20\end{array}$} & & & 0,60 & 0,17 & cde & \multirow{5}{*}{$\begin{array}{c}74,28 \pm \\
20,37\end{array}$} & & 58,48 & 16,77 & $d$ \\
\hline & \multirow{3}{*}{$\begin{array}{c}5.8 \\
(n=30)\end{array}$} & $5.3(n=10)$ & & & 20,07 & 3,41 & $a b c$ & & & & 0,69 & 0,15 & abcde & & & 72,01 & 14,25 & $\mathrm{~cd}$ \\
\hline & & $5.8(n=10)$ & & $\begin{array}{c}21,19 \pm \\
5,28\end{array}$ & 20,15 & 4,49 & $a b c$ & & $\begin{array}{c}0,14 \pm \\
0,20\end{array}$ & a & 0,72 & 0,23 & abcde & & $\begin{array}{c}99,3 / \pm \\
37,00\end{array}$ & 74,57 & 22,28 & $\mathrm{~cd}$ \\
\hline & & $6.3(n=10)$ & & & 22,35 & 4,98 & $a b$ & & & & 0,80 & 0,21 & abcd & & & 79,39 & 25,08 & bcd \\
\hline & \multirow{3}{*}{$\begin{array}{c}6.3 \\
(n=30)\end{array}$} & $6.8(n=10)$ & & \multirow{3}{*}{$\begin{array}{c}20,88 \pm \\
4,99\end{array}$} & 24,60 & 1,88 & $a$ & & \multirow{3}{*}{\multicolumn{2}{|c|}{$\begin{array}{c}0,71 \pm \\
0,19\end{array}$}} & 0,90 & 0,14 & a & & \multirow{3}{*}{$\begin{array}{l}98,65 \\
33,69\end{array}$} & 86,96 & 12,59 & $a b c d$ \\
\hline \multirow{5}{*}{$\begin{array}{c}\text { BAF } \\
(n=50)\end{array}$} & & $4.8(n=10)$ & \multirow{5}{*}{$\underset{3,74}{17,76 \pm}$ b } & & 15,39 & 3,08 & c & \multirow{5}{*}{0,13} & & & 0,50 & 0,11 & e & & & 84,16 & 31,22 & $a b c d$ \\
\hline & & $5.3(n=10)$ & & & 17,41 & 2,43 & $\mathrm{bc}$ & & & & 0,57 & 0,09 & de & & & 107,04 & 26,53 & $a b c$ \\
\hline & \multirow{3}{*}{$\begin{array}{c}6.8 \\
(n=30)\end{array}$} & $5.8(n=10)$ & & \multirow{3}{*}{$\underset{4,82}{19,98 \pm} a b$} & 20,22 & 3,90 & $a b c$ & & \multirow{3}{*}{\multicolumn{2}{|c|}{$\begin{array}{c}0,69 \pm \\
0,21\end{array}$}} & 0,67 & 0,14 & abcde & $\begin{array}{c}100,6 \pm \\
36,22\end{array}$ a & & 116,71 & 49,65 & $a b$ \\
\hline & & $6.3(n=10)$ & & & 20,00 & 4,12 & $a b c$ & & & & 0,66 & 0,15 & bcde & & $\begin{array}{c}95,15 \pm \\
22,32\end{array}$ & 120,30 & 36,45 & $a$ \\
\hline & & $6.8(n=10)$ & & & 15,80 & 2,39 & c & & & & 0,52 & 0,10 & e & & & 104,80 & 27,98 & $a b c$ \\
\hline
\end{tabular}

\section{Análisis de Suillus granulatus}

-A nivel de cepa

El análisis de los datos evidencia interacciones con diferencias significativas de los factores medio de cultivo y nivel de $\mathrm{pH}$ sobre las variables respuesta CR, VCM y B (cuadro 5).

Cuadro 5: Significancia estadística del análisis de varianza (ANDEVA) para los valores medios obtenidos de las variables crecimiento radial (CR), velocidad media de crecimiento (VMC) y biomasa (B) para cepas de la especie Suillus granulatus.

\begin{tabular}{|c|c|c|c|c|c|c|}
\hline \multirow[b]{2}{*}{ Factor } & \multicolumn{3}{|c|}{ Cepa IF937001 } & \multicolumn{3}{|c|}{ Cepa IF 1616002} \\
\hline & $\begin{array}{c}\text { CR } \\
(\mathrm{mm})\end{array}$ & $\begin{array}{c}\text { VMC } \\
\text { (mm/día) }\end{array}$ & $\begin{array}{c}\text { B } \\
(\mathrm{mg})\end{array}$ & $\begin{array}{c}\text { CR } \\
(\mathrm{mm})\end{array}$ & $\begin{array}{c}\text { VMC } \\
\text { (mm/día) }\end{array}$ & $B(\mathrm{mg})$ \\
\hline Medio & $0,0006^{\star}$ & $0,001^{*}$ & $<0,0001^{*}$ & $<0,0001^{*}$ & $<0,0001^{*}$ & $<0,0001^{*}$ \\
\hline $\mathrm{pH}$ & $<0,0001^{*}$ & $0,0001^{*}$ & $0,0002^{*}$ & $0,0005^{*}$ & $0,0071^{*}$ & $0,0001^{*}$ \\
\hline Medio $\times \mathrm{pH}$ & $0,0023^{*}$ & $0,0107^{*}$ & $0,0152^{*}$ & $0,0056^{*}$ & $0,0367^{*}$ & $0,0074^{*}$ \\
\hline
\end{tabular}

-Crecimiento Radial: Para la cepa IF937002, se obtuvo el mayor crecimiento radial en el medio PDA a pH 5,8 , aunque sin diferenciarse de los resultados obtenidos en medio PDA y BAF desde 5,3 a 6,3, y en MMN a pH 6,8. El crecimiento en medio MMN no fue influenciado por los diferentes niveles de $\mathrm{pH}$. En promedio, en todos los medios, el $\mathrm{pH}$ 4,8 tuvo menor crecimiento. A nivel de $\mathrm{pH}$, los valores de 5,8,6,3 y 5,3 lograron mayor desarrollo. A nivel de medios de cultivo, PDA y BAF presentaron los mayores crecimientos, diferenciándose significativamente de MMN. Por su parte, para la cepa IF1616002, se apreciaron los valores más altos en medios BAF y PDA, entre rangos de $\mathrm{pH}$ de 5,3 a 6,8 para el primero y de 5,3 a 5,8 más 6,8 para el segundo. Los valores significativamente menores fueron obtenidos en el medio MMN para $\mathrm{pH} 4,8$ y 5,3 . Se observó, además, un efecto nulo del $\mathrm{pH}$ en los crecimientos obtenidos en los medio MMN y PDA. Respecto al pH, los ajustes entre 5,3 y 6,8, generaron el mayor crecimiento radial, en tanto que, entre los medios de cultivo, el mejor resultado se obtuvo con BAF (cuadro 6).

- Velocidad Media de Crecimiento: Exhibió un comportamiento similar a la variable CR; el medio PDA a pH 5.8 presenta la mayor velocidad para la cepa IF937002, diferenciándose significativamente de los promedios obtenidos en $\mathrm{pH} 4,8$ y 6,8 para el mismo medio, en $\mathrm{BAF}$ a pH 4,8 y en $\mathrm{MMN}$ a pH entre 4,8 y 5,8 . En el medio MMN se observa un nulo efecto de los diferentes ajustes de $\mathrm{pH}$ en los resultados obtenidos. Respecto al pH se observa una mejor respuesta para valores entre 5,3 y 6,8, mientras que a nivel de medio de cultivo, los medios PDA y BAF son los que presentan crecimiento más rápido. Para la cepa IF1616002, el medio BAF a pH 5,8 permitió la mayor velocidad de crecimiento, pero el igual que la cepa anterior, no se diferencia de la velocidad obtenida en PDA y BAF con diferentes rangos de $\mathrm{pH}$. Se 
observó un nulo efecto de los diferentes ajustes de pH en los medios MMN y PDA y los crecimientos más lentos en MMN, y en $\mathrm{pH}$ 4,8 y 5,3.

A nivel de $\mathrm{pH}$, el mejor desempeño se observa para valores entre 5,3 y 6,8 . Para el factor medio de cultivo, los medios MMN y PDA presentaron los promedios más altos, diferenciándose significativamente con respecto al medio MMN (cuadro 6).

-Biomasa: En la cepa IF937002 el medio BAF a pH 5,8 exhibe la mayor producción de materia seca, sin diferenciarse significativamente de la producción obtenida con mismo medio a pH 5,3 y 6,3, ni con PDA a $\mathrm{pH}$ entre 5,3 y 6,3 . Los montos de materia seca más bajos se presentan en los tratamientos en base a MMN, como también para valores que fueron ajustados a $\mathrm{pH} \mathrm{4,8}$ en los tres medios utilizados. En los medios PDA y MMN el efecto de los diferentes ajustes de $\mathrm{pH}$ fue nulo. Respeto al $\mathrm{pH}$, los valores entre 5,3 y 6,3, son los más efectivos, siendo 5,8 el valor con mayor producción de biomasa. En cuanto a los medios de cultivo, PDA y BAF fueron significativamente mejores que MMN. La cepa IF1616002 presentó un comportamiento similar al de la cepa anterior: logra la mayor biomasa en el medio BAF a pH 5,8, y BAF a 6,3; la menor en todos los tratamientos del medio MMN y la menor absoluta en MMN a pH 4,8. También se observa un nulo efecto de los diferentes ajustes de $\mathrm{pH}$ en los medios PDA y MMN. Respecto al factor $\mathrm{pH}$, se la mayor biomasa se obtuvo para los ajustes entre 5,3 y 6,3. En cuanto a los medios de cultivo, BAF permite mayor producción de biomasa seca que los dos medios restantes (cuadro 6).

-Correlaciones entre Variables: En ambas cepas se observa una alta correlación entre CR y B (0,84 y $0,94)$ y entre VMC y $B(0,83$ y 0,95$)$.

Cuadro 6. Efecto del medio de cultivo y pH sobre los valores medios $(\mathrm{m})$ y desviaciones estándar (DE) de las variables crecimiento radial, velocidad media de crecimiento y producción de biomasa a nivel de cepas de Suillus granulatus.

\begin{tabular}{|c|c|c|c|c|c|c|c|c|c|c|c|c|c|c|c|c|c|}
\hline \multirow{3}{*}{ Cepa } & \multicolumn{2}{|c|}{ Factores } & \multirow{3}{*}{\begin{tabular}{|r|} 
Interacción \\
Medio*Ph
\end{tabular}} & \multicolumn{5}{|c|}{ Crecimiento radial $(\mathrm{mm})$} & \multicolumn{4}{|c|}{ Velocidad media de crecimiento (mm/día) } & \multicolumn{5}{|c|}{ Biomasa (mg) } \\
\hline & \multirow{2}{*}{ Medio } & \multirow{2}{*}{$\mathrm{pH}$} & & \multirow{2}{*}{$\begin{array}{l}\text { Medio } \\
\mathrm{m} \pm \mathrm{DE}\end{array}$} & $\mathrm{pH}$ & \multirow{2}{*}{\multicolumn{3}{|c|}{$\begin{array}{c}\text { Medio*Ph } \\
\mathrm{m} \pm \mathrm{DE}\end{array}$}} & \multirow{2}{*}{$\begin{array}{l}\text { Medio } \\
\mathrm{m} \pm \mathrm{DE} \\
\end{array}$} & \multirow{2}{*}{$\begin{array}{c}\mathrm{pH} \\
\mathrm{m} \pm \mathrm{DE}\end{array}$} & \multirow{2}{*}{\multicolumn{2}{|c|}{$\begin{array}{c}\text { Medio*Ph } \\
\mathrm{m} \pm \mathrm{DE}\end{array}$}} & \multirow{2}{*}{$\begin{array}{c}\text { Medio } \\
\mathrm{m} \pm \mathrm{DE}\end{array}$} & \multirow{2}{*}{$\begin{array}{c}\mathrm{pH} \\
\mathrm{m} \pm \mathrm{DE}\end{array}$} & \multicolumn{3}{|c|}{ Medio*Ph } \\
\hline & & & & & $\mathrm{m} \pm \mathrm{DE}$ & & & & & & & & & & & $m \pm D E$ & \\
\hline \multirow{15}{*}{$\begin{array}{l}\text { IF } 937002 \\
(n=75)\end{array}$} & \multirow{5}{*}{$\begin{array}{c}\text { PDA } \\
(n=25)\end{array}$} & \multirow{3}{*}{$\begin{array}{c}4.8 \\
(n=15)\end{array}$} & $4.8(n=5)$ & \multirow{5}{*}{$\begin{array}{c}23,05 \pm \\
5,24\end{array}$ a } & \multirow{3}{*}{$\begin{array}{c}18,41 \pm \\
2,86\end{array}$} & 18,59 & 2,74 & d & \multirow{5}{*}{$\begin{array}{c}0,76 \pm \\
0,17\end{array}$} & \multirow{3}{*}{$\begin{array}{c}0,62 \pm \\
0,11\end{array}$} & 0,62 & $0,08 \mathrm{c}$ & \multirow{5}{*}{$\begin{array}{c}120,74 \pm \\
33,82\end{array}$} & & 88,56 & 10,28 & bcde \\
\hline & & & $5.3(n=5)$ & & & 26,91 & 4,04 & $a b$ & & & 0,89 & $0,14 \mathrm{ab}$ & & $\begin{array}{c}69,92 \pm c \\
25,34\end{array}$ & 141,81 & 25,54 & $a b$ \\
\hline & & & $5.8(n=5)$ & & & 28,09 & 4,89 & $a$ & & & 0,93 & 0,14 a & & & 138,70 & 36,29 & $a b$ \\
\hline & & & $6.3(n=5)$ & & & 21,63 & 4,68 & abcd & & & 0,70 & 0,16 abc & & & 131,44 & 41,81 & $a b c$ \\
\hline & & $\begin{array}{c}5.3 \\
(n=15)\end{array}$ & $6.8(n=5)$ & & $23,07 \pm a b$ & 20,04 & 2,50 & bcd & & $0,78 \pm$ & 0,68 & 0,08 bc & & $100,31 \pm a$ & 103,18 & 16,14 & bcde \\
\hline & & & $4.8(n=5)$ & & & 17,32 & 1,23 & d & & & 0,58 & $0,07 \mathrm{c}$ & & & 41,42 & 10,96 & $\mathrm{e}$ \\
\hline & & & $5.3(n=5)$ & & & 19,64 & 1,34 & $\mathrm{~cd}$ & & & 0,67 & $0,04 \mathrm{bc}$ & & & 49,58 & 16,95 & de \\
\hline & $(n=25)$ & $\begin{array}{c}5.8 \\
(n=15)\end{array}$ & $5.8(n=5)$ & $\begin{array}{c}19,61 \pm b \\
2,28\end{array}$ & $\begin{array}{c}24,32 \pm \\
5,64\end{array}$ & 18,27 & 2,02 & d & $\begin{array}{c}0,60 \pm \\
0,09\end{array}$ & $\begin{array}{c}0,82 \pm \\
0,17\end{array}$ a & 0,63 & $0,06 \mathrm{c}$ & $\begin{array}{c}57,36 \pm \\
22,20\end{array}$ b & $\begin{array}{c}120,19 \pm \\
64,58\end{array}$ & 49,84 & 6,21 & de \\
\hline & & & $6.3(n=5)$ & & & 20,90 & 2,22 & bcd & & & 0,68 & 0,12 abc & & & 77,70 & 36,34 & bcde \\
\hline & & & $6.8(n=5)$ & & & 21,92 & 1,14 & abcd & & & 0,75 & $0,07 \quad a b c$ & & & 68,24 & 7,84 & cde \\
\hline & & $\begin{array}{c}6.3 \\
(n=15)\end{array}$ & $4.8(n=5)$ & & $\underset{3,35}{21,88 \pm} \mathrm{ab}$ & 19,30 & 4,13 & d & & $\begin{array}{c}0,73 \pm \\
0,14\end{array}$ & 0,66 & 0,15 bc & & $\begin{array}{c}108,42 \pm a \\
43,46\end{array}$ & 79,78 & 21,24 & bcde \\
\hline & & & $5.3(n=5)$ & & & 22,67 & 3,59 & abcd & & & 0,79 & $0,13 a b c$ & & & 109,54 & 27,61 & $a b c d$ \\
\hline & $\begin{array}{c}\text { BAt } \\
(n=25)\end{array}$ & & $5.8(n=5)$ & $\begin{array}{c}22,45 \pm \\
405\end{array}$ & & 26,60 & 3,68 & $a b c$ & $0,78 \pm$ & & 0,91 & $0,11 \mathrm{ab}$ & $\begin{array}{c}115,36 \pm \text { a } \\
47,46\end{array}$ & & 173,84 & 55,03 & $a$ \\
\hline & & $\begin{array}{c}6.8 \\
(n=15)\end{array}$ & $6.3(n=5)$ & & $20,84 \pm b c$ & 23,12 & 3,05 & abcd & & $0,72 \pm \quad a b$ & 0,80 & $0,14 a b c$ & & $89,65 \pm$ bc & 116,12 & 40,32 & $a b c$ \\
\hline & & & $6.8(n=5)$ & & & 20,55 & 2,52 & bcd & & & 0,73 & 0,09 abc & & & 97,54 & 36,76 & bcde \\
\hline & & & $4.8(n=5)$ & & & 19,45 & 3,91 & abcd & & & 0,66 & $0,11 \mathrm{ab}$ & & & 75,64 & 14,44 & cde \\
\hline & & $\begin{array}{c}4.8 \\
(n=15)\end{array}$ & $5.3(n=5)$ & & $\begin{array}{c}15,97 \pm \quad b \\
4,71\end{array}$ & 22,83 & 1,33 & $a b$ & & $0,54 \pm \quad b$ & 0,77 & $0,03 \mathrm{ab}$ & & $\begin{array}{c}63,25 \pm \\
27,16\end{array}$ & 104,86 & 15,49 & $b c$ \\
\hline & $(n=25)$ & & $5.8(n=5)$ & $\begin{array}{l}20,5 \pm \\
287\end{array}$ & & 20,69 & 2,53 & $a b$ & $\begin{array}{c}0,69 \pm \\
0,09\end{array}$ & & 0,71 & $0,10 \mathrm{ab}$ & $\begin{array}{c}94,39 \pm b \\
16,50\end{array}$ & & 94,90 & 11,83 & $b c$ \\
\hline & & & $6.3(n=5)$ & & & 18,74 & 2,82 & bcd & & & 0,64 & $0,09 a b c$ & & & 96,98 & 13,93 & bc \\
\hline & & $\begin{array}{c}5.3 \\
(n=15)\end{array}$ & $6.8(n=5)$ & & $17,81 \pm a b$ & 19,77 & 2,32 & $a b c$ & & $0,61 \pm a b$ & 0,68 & $0,07 \quad a b$ & & $82,8 / \pm$ & 99,56 & 15,26 & $b c$ \\
\hline & & & $4.8(n=5)$ & & & 10,56 & 0,53 & $f$ & & & 0,35 & 0,02 ef & & & 31,38 & 1,64 & $f$ \\
\hline & & & $5.3(n=5)$ & & & 10,49 & 0,70 & $f$ & & & 0,34 & 0,03 ef & & & 31,86 & 2,45 & $f$ \\
\hline $\begin{array}{l}\text { IF1616002 } \\
(\mathrm{n}=75)\end{array}$ & $\begin{array}{l}\text { MMN } \\
(n=25)\end{array}$ & $\begin{array}{c}5.8 \\
(n=15)\end{array}$ & $5.8(n=5)$ & $\begin{array}{c}12,82 \pm b \\
232\end{array}$ & $\begin{array}{c}19,92 \pm \\
4,20\end{array}$ & 15,17 & 2,22 & cdef & $0,41 \pm$ & $0,66 \pm$ a & 0,46 & 0,09 cdef & $\begin{array}{l}38,8 \pm \\
10,28\end{array}$ & $\begin{array}{c}95,43 \pm \\
48,06\end{array}$ & 40,12 & 13,15 & ef \\
\hline & $(n=25)$ & & $6.3(n=5)$ & & & 13,48 & 1,15 & ef & & & 0,45 & 0,04 def & & & 46,94 & 7,63 & def \\
\hline & & & $6.8(n=5)$ & & & 14,42 & 1,26 & def & & & 0,46 & 0,07 cdef & & & 43,68 & 12,24 & ef \\
\hline & & $\begin{array}{c}0.3 \\
(n=15)\end{array}$ & $4.8(n=5)$ & & $\begin{array}{c}17,75 \pm \\
4,08\end{array}$ & 17,90 & 2,39 & bcde & & $\begin{array}{c}0,00 \pm \\
0,16\end{array}$ & 0,62 & 0,09 bcd & & $\begin{array}{c}81,18 \pm \\
38,59\end{array}$ & 82,72 & 20,87 & bcd \\
\hline & & & $5.3(n=5)$ & & & 20,94 & 3,01 & $a b$ & & & 0,71 & $0,11 \mathrm{ab}$ & & & 111,90 & 22,16 & $b c$ \\
\hline & $\begin{array}{l}\text { BAF } \\
(n=25)\end{array}$ & & $5.8(n=5)$ & $\begin{array}{c}20,33 \pm \quad a \\
3,04\end{array}$ & $1790+$ & 23,90 & 1,26 & $a$ & $0,11 \pm$ & $060+$ & 0,82 & $0,07 \quad a$ & $\begin{array}{c}113,24 \pm \\
31,36\end{array}$ & & 151,28 & 6,87 & $a$ \\
\hline & & $\begin{array}{c}6.8 \\
(n=15)\end{array}$ & $6.3(n=5)$ & & $\frac{17,90 \pm}{3,06} \mathrm{ab}$ & 21,02 & 3,41 & $a b$ & & $\begin{array}{c}0,60 \pm \\
0,12\end{array} a b$ & 0,72 & $0,17 \mathrm{ab}$ & & $\begin{array}{l}85,3 \pm \\
33,95\end{array}$ & 117,62 & 40,71 & $a b$ \\
\hline & & & $6.8(n=5)$ & & & 19,53 & 1,72 & $a b c$ & & & 0,67 & $0,05 a b$ & & & 112,67 & 17,19 & bc \\
\hline
\end{tabular}

\section{-A Nivel de Especie}

-Crecimiento Radial: Los mejores crecimientos fueron obtenidos en los medios BAF y PDA con pH entre 5,3 y 6,8, siendo los mayores absolutos los obtenidos con PDA a pH 5,3 y BAF a pH 5,8. No se observa efecto del factor $\mathrm{pH}$ en el medio MMN, el cual exhibe el menor crecimiento, particularmente con $\mathrm{pH} 4,8$. Las mejores respuestas al pH, se presentan para el rango 5,3 a 6,3 (cuadro 7). 
- Velocidad Media de Crecimiento: La mayor velocidad se obtuvo en medio BAF a pH 5,8, pero sin diferenciarse respecto a BAF a pH 5,3, 6,3 y 6,8, ni respecto a PDA con pH entre 5,3 y 6,8. En tanto que, el medio MMN, presentó los promedios de velocidades más bajas, particularmente con pH 4,8. Para el factor $\mathrm{pH}$, los valores entre 5,3 y 6,8, presentaron los mejores desempeños, siendo los ajustes a 5,8 y 5,3 los que logran los valores absolutos más altos. Para el factor medio de cultivo, los mejores promedios fueron para PDA y BAF, diferenciándose significativamente del medio MMN (cuadro 7).

-Biomasa: La mayor biomasa se obtuvo en el medio BAF a pH 5,8, con diferencias significativas con el resto de los tratamientos. El medio MMN presenta los valores promedios más bajos y sin efecto de los valores de $\mathrm{pH}$. Para el factor $\mathrm{pH}$, valores entre 5,3 y 6,3 presentan buenos desempeños y no se diferencian estadísticamente entre sí, siendo 5,8 el pH que genera la mayor producción de biomasa. Respecto a los medios de cultivo, PDA Y BAF, presentaron los mayores montos de materia seca diferenciándose significativamente del medio MMN (cuadro 7).

-Correlaciones entre Variables: La correlación entre las variables CR y B y entre VMC y B son altas $(0,94$ y 0,95 respectivamente).

Cuadro 7. Efecto del medio de cultivo y $\mathrm{pH}$ sobre los valores medios $(\mathrm{m})$ y desviaciones estándar (DE) de las variables crecimiento radial, velocidad media de crecimiento y producción de biomasa para Suillus granulatus.

\begin{tabular}{|c|c|c|c|c|c|c|c|c|c|c|c|c|c|c|c|c|c|c|c|c|}
\hline \multicolumn{2}{|c|}{ Factores } & \multirow{2}{*}{$\begin{array}{l}\text { Interacción } \\
\text { Medio }^{*} \mathrm{Ph}\end{array}$} & \multicolumn{5}{|c|}{ Crecimiento radial $(\mathrm{mm})$} & \multicolumn{7}{|c|}{ Velocidad media de crecimiento (mm/día) } & \multicolumn{6}{|c|}{ Biomasa (mg) } \\
\hline Medio & $\mathrm{pH}$ & & $\begin{array}{c}\text { Medio } \\
\mathrm{m} \pm \mathrm{DE}\end{array}$ & $\begin{array}{c}\mathrm{pH} \\
\mathrm{m} \pm \mathrm{DE}\end{array}$ & & $\begin{array}{l}\text { edio* }^{*} p \\
m \pm D E\end{array}$ & & $\begin{array}{r}\text { Medio } \\
\mathrm{m} \pm \mathrm{DE}\end{array}$ & & $\begin{array}{r}\mathrm{ph} \\
\mathrm{m} \pm\end{array}$ & & & $\begin{array}{l}\text { hedio* } \\
m \pm D E\end{array}$ & & $\begin{array}{r}\text { Medio } \\
\mathrm{m} \pm \mathrm{DE}\end{array}$ & $\begin{array}{c}\mathrm{pH} \\
\mathrm{m} \pm \mathrm{DE}\end{array}$ & & & $\begin{array}{c}\text { Medio*p } \\
\mathrm{m} \pm \mathrm{DE}\end{array}$ & \\
\hline \multirow{5}{*}{$\begin{array}{c}\text { PDA } \\
(n=50)\end{array}$} & \multirow{3}{*}{$\begin{array}{c}4.8 \\
(n=30)\end{array}$} & $4.8(n=10)$ & \multirow{5}{*}{$\begin{array}{c}21,68 \pm \\
4,41\end{array} a$} & \multirow{3}{*}{$\underset{4,02}{17,19 \pm} \mathrm{c}$} & 19,02 & 3,22 & bcde & \multirow{5}{*}{$\begin{array}{c}0,73 \pm \\
0,14\end{array}$} & \multirow{5}{*}{ a } & \multirow{3}{*}{$\begin{array}{c}0,58 \pm \\
0,14\end{array}$} & \multirow{3}{*}{ b } & 0,64 & 0,10 & bcde & \multirow{5}{*}{$\begin{array}{c}107,56 \pm \\
29,51\end{array}$} & \multirow{3}{*}{$66,58 \mathrm{c}$} & \multirow{3}{*}{ c } & 82,10 & 13,64 & $\mathrm{~cd}$ \\
\hline & & $5.3(n=10)$ & & & 24,87 & 3,56 & a & & & & & 0,83 & 0,12 & $a b$ & & & & 123,33 & 27,85 & $b$ \\
\hline & & $5.8(n=10)$ & & & 24,39 & 5,35 & $a b$ & & & & & 0,82 & 0,16 & $a b$ & & & & 116,80 & 34,36 & $\mathrm{bc}$ \\
\hline & \multirow{3}{*}{$\begin{array}{c}5.3 \\
(n=30)\end{array}$} & $6.3(n=10)$ & & \multirow{3}{*}{$\begin{array}{c}20,47 \pm \\
-5,66\end{array} a b$} & 20,19 & 3,95 & abcd & & & \multirow{3}{*}{$\begin{array}{c}0,69 \pm \\
0,20\end{array}$} & \multirow{3}{*}{ a } & 0,67 & 0,13 & abcd & & \multirow{3}{*}{91,59} & & 114,21 & 34,54 & bc \\
\hline & & $6.8(n=10)$ & & & 19,91 & 2,28 & abcd & & & & & 0,68 & 0,07 & abcd & & & $a b$ & 101,37 & 14,93 & $b c$ \\
\hline \multirow{5}{*}{$\begin{array}{l}\text { MMN } \\
(n=50)\end{array}$} & & $4.8(n=10)$ & \multirow{5}{*}{$\begin{array}{c}16,22 \pm \\
4,11\end{array}$} & & 13,94 & 3,67 & e & \multirow{5}{*}{$\begin{array}{c}0,54 \pm \\
0,15\end{array}$} & & & & 0,46 & 0,13 & $\mathrm{e}$ & & & & 36,40 & 9,09 & e \\
\hline & \multirow{3}{*}{$\begin{array}{c}5.8 \\
(n=30)\end{array}$} & $5.3(n=10)$ & & & 15,07 & 4,93 & de & & \multirow{4}{*}{$b$} & \multirow{3}{*}{$\begin{array}{c}0,74 \pm \\
0,19\end{array}$} & \multirow{3}{*}{ a } & 0,50 & 0,18 & de & & & & 40,72 & 14,75 & e \\
\hline & & $5.8(n=10)$ & & $537 a$ & 16,72 & 2,59 & cde & & & & & 0,55 & 0,11 & de & 48,08I b & 108,11 & a & 44,98 & 10,97 & de \\
\hline & & $6.3(n=10)$ & & & 17,19 & 4,25 & cde & & & & & 0,56 & 0,15 & cde & & & & 62,32 & 29,59 & de \\
\hline & & $6.8(n=10)$ & & & 18,17 & 4,11 & cde & & & & & 0,61 & 0,17 & cde & & & & 55,96 & 16,17 & de \\
\hline & $(n=30)$ & $4.8(n=10)$ & & $19,81 \pm a b c$ & 18,60 & 3,27 & cde & & & $0,66 \pm$ & $a b$ & 0,64 & 0,12 & bcde & & 97,80 & $a b$ & 81,25 & 19,91 & $\mathrm{~cd}$ \\
\hline & & $5.3(n=10)$ & & & 21,48 & 3,37 & $a b c$ & & & & & 0,75 & 0,12 & $a b c$ & & & & 110,72 & 23,63 & bc \\
\hline$(n=50)$ & 68 & $5.8(n=10)$ & $\left.\right|_{3,67} ^{21,49 \pm} a$ & $19.37+$ & 25,25 & 2,96 & $a$ & $0,14 \pm$ & a & $0.66+$ & & 0,86 & 0,10 & $a$ & $\underset{39,81}{110,3 \pm}$ a & & & 162,56 & 38,84 & a \\
\hline & $(n=30)$ & $6.3(n=10)$ & & $\underset{3,00}{19,3 / \pm} \mathrm{bc}$ & 22,07 & 3,24 & $a b c$ & & & $\begin{array}{l}0,00 \pm \\
0,12\end{array}$ & $a b$ & 0,76 & 0,15 & $a b c$ & & 87,48 & b & 116,87 & 38,21 & bc \\
\hline & & $6.8(n=10)$ & & & 20,04 & 2,11 & abcd & & & & & 0,70 & 0,08 & abcd & & & & 105,11 & 28,21 & bc \\
\hline
\end{tabular}

\section{Análisis de Suillus bellinii}

\section{-A Nivel de Cepa}

El análisis de los datos obtenidos para las cepas IF713001 y IF633001 evidencia interacciones con significativa estadística entre los factores $\mathrm{pH}$ y medio de cultivo sobre las variables respuesta CR, VMC y B (cuadro 8).

Cuadro 8. Significancia estadística del análisis de varianza (ANDEVA) para los valores medios obtenidos de las variables crecimiento radial (CR), velocidad media de crecimiento (VMC) y biomasa $(\mathrm{B})$.

\begin{tabular}{ccccccc}
\hline \multirow{2}{*}{ Factor } & \multicolumn{3}{c}{ Cepa IF713001 } & \multicolumn{3}{c}{ Cepa IF 633001 } \\
\cline { 2 - 7 } & $\begin{array}{c}\mathbf{C R} \\
(\mathbf{m m})\end{array}$ & $\begin{array}{c}\text { VMC } \\
(\mathbf{m m} / \mathbf{d i ́})\end{array}$ & $\begin{array}{c}\text { B } \\
(\mathbf{m g})\end{array}$ & $\begin{array}{c}\text { CR } \\
(\mathbf{m m})\end{array}$ & $\begin{array}{c}\text { VMC } \\
(\mathbf{m m} / \mathbf{d i ́})\end{array}$ & $\begin{array}{c}\text { B } \\
(\mathbf{m g})\end{array}$ \\
\hline Medio & 0,2121 & $0,0182^{*}$ & $<0,0001^{*}$ & $0,0043^{*}$ & $0,0082^{*}$ & $<0,0001^{*}$ \\
\hline $\mathrm{pH}$ & $0,0288^{*}$ & $0,0330^{*}$ & 0,0903 & 0,3518 & 0,2623 & $0,0001^{*}$ \\
\hline Medio $\times \mathrm{pH}$ & $0,0146^{*}$ & $0,0042^{*}$ & $0,0071^{*}$ & $0,0179^{*}$ & $0,0297^{*}$ & $0,0021^{*}$ \\
\hline & & ${ }^{*}$ Efectos significativos $(P \leq 005)$ & &
\end{tabular}


-Crecimiento Radial: La cepa IF713001 presentó crecimientos bastante homogéneos para las distintas combinaciones de medio de cultivo y $\mathrm{pH}$, solo se diferenciaros significativamente los valores más altos, correspondientes a BAF y PDA a pH 5,8 y $\mathrm{MMN}$ a pH 6,8, respecto del más bajo, obtenido con MMN a $\mathrm{pH} 5,3$. No se observó efecto de los diferentes ajustes de $\mathrm{pH}$ en los medios BAF y PDA. Para el factor pH el mejor crecimiento se obtuvo con 5,8, pero sin diferencias respecto a 6,3 y 6,8 .

Respecto a los medios de cultivo, no hubo diferencias significativas entre ellos.

En cuanto a la cepa IF633001, esta presentó su mayor crecimiento en medio MMN a pH 6,8, pero diferenciándose solo del crecimiento obtenido con BAF a pH 6,3 y 6,8 y con el medio PDA a pH 5,3 y 6,8.

Se observó al igual que la cepa anterior, un nulo efecto de los diferentes ajustes de $\mathrm{pH}$ sobre los medios BAF y PDA. Para el factor $\mathrm{pH}$ no hubo diferencias significativas entre los diferentes ajustes evaluados. En tanto que, para el factor medio de cultivo, el medio MMN presentó el mayor crecimiento promedio, con diferencias significativas con los medios PDA y BAF (cuadro 9).

- Velocidad Media de Crecimiento: Para la cepa IF713001 la mayor velocidad se obtuvo en el medio PDA a pH 5,8, aunque diferenciándose estadísticamente sólo con $\mathrm{MMN}$ a pH 5,3 y 4,8. Se observó, al igual que en la variable $\mathrm{CR}$, un nulo efecto de los diferentes valores de $\mathrm{pH}$ sobre los medios BAF y PDA.

Para el factor $\mathrm{pH}$, la mayor velocidad se obtuvo a $\mathrm{pH} 5,8$, pero sin diferencias significativas con los promedios obtenidos para valores de 5,3,6,3 y 6,8. En tanto que, para el factor medio de cultivo, el valor mayor se obtuvo en PDA, pero sin diferencias significativas con el medio BAF.

En relación a la cepa IF633001, el tratamiento con mejor desempeño fue MMN a pH 6.8 con diferencias significativas con los medios BAF para valores de $\mathrm{pH}$ de 6,3 y 6,8, y PDA para pH de 5,3 y 6,8. Se presentan nulos efectos de los distintos niveles de $\mathrm{pH}$ en los tres medios de cultivo.

Para el factor $\mathrm{pH}$ no hubo diferencias significativas entre los diferentes valores de $\mathrm{pH}$. En tanto que, para el factor medio de cultivo, el medio MMN presentó la mayor velocidad promedio, con diferencias significativas con los medios BAF y PDA (cuadro 9).

-Biomasa: En la cepa IF713001 se observó una producción mayor de materia seca bajo el medio BAF a $\mathrm{pH} 5,3$ y 5,8, presentando diferencias significativas con los promedios obtenidos en los medios PDA, MMN y BAF a pH 6,8. La menor producción de biomasa seca se obtiene en MMN. Se apreció un nulo efecto de los distintos niveles de $\mathrm{pH}$ en los medios PDA y MMN.

Para el factor $\mathrm{pH}$ no hubo diferencias significativas entre los diferentes ajustes analizados. En tanto que, para el factor medio de cultivo, se obtuvo la mayor cantidad de peso seco en el medio BAF con diferencias significativas con los medios MMN y PDA.

En cuanto a la cepa IF633001, esta presenta buenos desempeños en biomasa para los medios BAF y PDA con valores de pH entre 4,8 a 5,8. Se observa un crecimiento menor en todos los tratamientos que contemplaron el medio MMN, como también, un nulo efecto de los distintos niveles de pH en los medios PDA y MMN.

Para el factor $\mathrm{pH}$, se obtuvo la mayor biomasa seca con 4,8 y 5,8, pero sin diferencias significativas con $\mathrm{pH}$ 5,3. Respecto a los medios de cultivo, la mayor biomasa seca se obtuvo con PDA y BAF (cuadro 9).

-Correlaciones entre variables: La correlación entre la biomasa seca y las variables de crecimiento exhibió un comportamiento muy diferente entre cepas. En IF713001 las correlaciones B-CR y B-VMC fueron de 0,50 y 0,44 respectivamente, en tanto que en IF633001 fueron de -0,02 y -0,03. 
Cuadro 9. Efecto del medio de cultivo y $\mathrm{pH}$ sobre los valores medios $(\mathrm{m})$ y desviaciones estándar (DE) de las variables crecimiento radial, velocidad media de crecimiento y producción de biomasa a nivel de cepas de Suillus bellinii.

\begin{tabular}{|c|c|c|c|c|c|c|c|c|c|c|c|c|c|c|c|c|c|}
\hline \multirow{3}{*}{ Cepa } & \multicolumn{2}{|c|}{ Factores } & \multirow{3}{*}{$\begin{array}{l}\text { Interacción } \\
\text { Medio*Ph }\end{array}$} & \multicolumn{5}{|c|}{ Crecimiento radial (mm) } & \multicolumn{4}{|c|}{ Velocidad media de crecimiento (mm/día) } & \multicolumn{5}{|c|}{ Biomasa (mg) } \\
\hline & \multirow{2}{*}{ Medio } & \multirow{2}{*}{$\mathrm{pH}$} & & Medio & $\mathrm{pH}$ & & Medio*Ph & & Medio & $\mathrm{pH}$ & & edio* $\mathrm{Ph}$ & Medio & $\mathrm{pH}$ & & edio*Ph & \\
\hline & & & & $\mathrm{m} \pm \mathrm{DE}$ & $\mathrm{m} \pm \mathrm{DE}$ & & $\mathrm{m} \pm \mathrm{DE}$ & & $\mathrm{m} \pm \mathrm{DE}$ & $\mathrm{m} \pm \mathrm{DE}$ & & $\mathrm{m} \pm \mathrm{DE}$ & $\mathrm{m} \pm \mathrm{DE}$ & $\mathrm{m} \pm \mathrm{DE}$ & & $m \pm D E$ & \\
\hline \multirow{15}{*}{$\begin{array}{c}\text { IF } 713001 \\
(n=75)\end{array}$} & \multirow{5}{*}{$\begin{array}{c}\text { PDA } \\
(n=25)\end{array}$} & \multirow{3}{*}{$\begin{array}{c}4.8 \\
(n=15)\end{array}$} & $4.8(n=5)$ & \multirow{5}{*}{$\begin{array}{c}18,18 \pm \\
2,18\end{array} \quad$ a } & \multirow{3}{*}{$\underset{2,25}{16,97 \pm} \mathrm{b}$} & 17,36 & $1,55 \mathrm{a}$ & $a b$ & \multirow{5}{*}{$\begin{array}{l}0,65 \pm \\
0,07\end{array}$} & \multirow{3}{*}{$\begin{array}{c}0,59 \pm \\
0,08\end{array}$} & 0,62 & $0,06 a b c$ & \multirow{5}{*}{$\begin{array}{c}80,49 \pm \\
12,45\end{array}$} & \multirow{3}{*}{$\underset{33,65}{80,77 \pm}$} & 78,06 & 7,99 & def \\
\hline & & & $5.3(n=5)$ & & & 17,85 & 2,31 & $a b$ & & & 0,65 & $0,08 a b c$ & & & 84,90 & 9,48 & bcde \\
\hline & & & $5.8(n=5)$ & & & 19,71 & 1,96 & $a$ & & & 0,70 & 0,08 a & & & 85,85 & 10,80 & bcd \\
\hline & & \multirow{3}{*}{$\begin{array}{c}5.3 \\
(n=15)\end{array}$} & $6.3(n=5)$ & & \multirow{3}{*}{$\begin{array}{c}17,02 \pm \\
2,74\end{array}$} & 18,38 & $2,05 \quad a$ & $a b$ & & \multirow{3}{*}{$\begin{array}{c}0,60 \pm \\
0,10\end{array} a b$} & 0,66 & $0,06 a b c$ & & \multirow{3}{*}{$\begin{array}{c}85,91 \pm \\
37,87\end{array}$} & 80,84 & 13,90 & cde \\
\hline & & & $6.8(n=5)$ & & & 17,59 & $2,89 \quad$ & $a b$ & & & 0,63 & $0,08 a b c$ & & & 72,83 & 18,05 & def \\
\hline & \multirow{5}{*}{$\begin{array}{l}\text { MMN } \\
(n=25)\end{array}$} & & $4.8(n=5)$ & \multirow{5}{*}{$\begin{array}{c}17,13 \pm \\
3,11\end{array}$} & & 15,05 & $0,90=$ & $a b$ & & & 0,51 & 0,03 bc & & & 48,92 & 5,86 & ef \\
\hline & & & $5.3(n=5)$ & & & 14,39 & 2,38 & b & & & 0,49 & $0,07 \mathrm{c}$ & & & 43,84 & 8,05 & $f$ \\
\hline & & $\begin{array}{c}5.8 \\
(n=15)\end{array}$ & $5.8(n=5)$ & & $19,48 \pm$ a & 18,60 & $4,13 \quad$ & $a b$ & $0,59 \pm$ & $0,0 / \pm \quad$ a & 0,64 & $0,13 a b c$ & $53,98 \pm$ & $89,68 \pm$ a & 58,84 & 13,26 & def \\
\hline & & & $6.3(n=5)$ & & & 18,08 & 1,64 & $a b$ & & & 0,62 & $0,06 a b c$ & & & 53,38 & 4,08 & def \\
\hline & & 63 & $6.8(n=5)$ & & & 19,55 & 2,43 & $a$ & & & 0,68 & $0,08 a b$ & & & 64,90 & 12,30 & def \\
\hline & & $(n=15)$ & $4.8(n=5)$ & & $17,09 \pm a b$ & 18,51 & 2,60 & $a b$ & & $0,02 \pm \quad a b$ & 0,64 & $0,08 a b c$ & & $84,0 \pm$ & 115,34 & 33,07 & $a b c$ \\
\hline & & & $5.3(n=5)$ & & & 18,83 & $1,33 \quad$ & $a b$ & & & 0,65 & $0,05 a b c$ & & & 128,98 & 18,26 & a \\
\hline & $(n=25)$ & 68 & $5.8(n=5)$ & $\begin{array}{c}10,1 \pm \quad a \\
2,48\end{array}$ & $1767+$ & 20,12 & 3,35 & a & $0,02 \pm a b$ & $0.62+$ & 0,67 & $0,11 \mathrm{ab}$ & $114,23 \pm a$ & $7357+$ & 124,36 & 27,18 & $a$ \\
\hline & & $\begin{array}{l}0.8 \\
(n=15)\end{array}$ & $6.3(n=5)$ & & $17,01 \pm a b$ & 17,20 & 0,74 & $a b$ & & $0,02 \pm \quad a b$ & 0,59 & $0,02 a b c$ & & $15,31 \pm$ & 119,58 & 16,16 & $a b$ \\
\hline & & & $6.8(n=5)$ & & & 15,86 & $1,80 \mathrm{a}$ & $a b$ & & & 0,55 & 0,07 abc & & & 82,98 & 17,91 & cde \\
\hline & & & $4.8(n=5)$ & & & 19,58 & 2,52 & $a b$ & & & 0,66 & $0,09 a b$ & & & 111,56 & 4,51 & abcd \\
\hline & & $\begin{array}{c}4.0 \\
(n=15)\end{array}$ & $5.3(n=5)$ & & 231 a & 18,53 & 4,05 & $\mathrm{~b}$ & & 0,001 & 0,59 & $0,12 \mathrm{~b}$ & & $\begin{array}{c}11,401 \\
35,31\end{array}$ & 116,76 & 16,00 & $a b c$ \\
\hline & $(n=25)$ & & $5.8(n=5)$ & $\begin{array}{c}19,40 \pm \\
281\end{array}$ & & 22,01 & 2,81 & $a b$ & $0,03 \pm b$ & & 0,76 & $0,10 a b$ & $101,12 \pm a$ & & 124,76 & 16,67 & $a b c$ \\
\hline & & & $6.3(n=5)$ & & & 19,02 & 1,36 & $a b$ & & & 0,64 & $0,05 \mathrm{ab}$ & & & 88,20 & 8,11 & bcde \\
\hline & & $(n=15)$ & $6.8(n=5)$ & & 402 a & 18,15 & 1,83 & $\mathrm{~b}$ & & $0,14 \quad a$ & 0,60 & $0,06 \mathrm{~b}$ & & $33,92 b$ & 94,70 & 20,82 & bcde \\
\hline & & & $4.8(n=5)$ & & & 19,48 & 1,46 & $a b$ & & & 0,66 & $0,06 a b$ & & & 73,32 & 7,92 & de \\
\hline & & & $5.3(n=5)$ & & & 20,58 & 5,91 & $a b$ & & & 0,68 & $0,22 a b$ & & & 68,82 & 16,99 & e \\
\hline$(n=75)$ & $(n=25)$ & $(n=15)$ & $5.8(n=5)$ & $\begin{array}{c}21,04 \pm \\
4.50\end{array}$ & $\begin{array}{c}21,4 / \pm \\
381\end{array}$ & 22,58 & 5,02 & $a b$ & $0,14 \pm \quad a$ & 0,14 a & 0,76 & $0,20 a b$ & $10,35 \pm b$ & $\begin{array}{c}112,01 \pm a \\
31,31\end{array}$ & 84,28 & 18,90 & cde \\
\hline & & & $6.3(n=5)$ & & & 20,62 & $3,37 \quad$ & $a b$ & & & 0,69 & $0,14 a b$ & & & 71,12 & 10,81 & de \\
\hline & & 63 & $6.8(n=5)$ & & & 25,92 & 3,94 & $a$ & & & 0,88 & 0,15 a & & & 84,22 & 11,40 & cde \\
\hline & & $(n=15)$ & $4.8(n=5)$ & & 261 a & 21,14 & $2,83 \quad \mathrm{a}$ & $a b$ & & $\begin{array}{c}0,041 \\
0,10\end{array}$ & 0,71 & $0,10 a b$ & & $1774 \quad c$ & 149,50 & 25,56 & a \\
\hline & BAF & & $5.3(n=5)$ & & & 19,75 & $1,62 \quad \mathrm{a}$ & $a b$ & & & 0,65 & $0,05 \mathrm{ab}$ & & & 139,38 & 15,65 & $a$ \\
\hline & $(n=25)$ & 68 & $5.8(n=5)$ & $307 \quad b$ & $1996+$ & 19,83 & 3,52 & $a b$ & 010 b & $067+$ & 0,67 & $0,11 a b$ & $3396 \quad a$ & $8953+$ & 126,98 & 36,77 & $a b$ \\
\hline & & $\begin{array}{c}0.0 \\
(n=15)\end{array}$ & $6.3(n=5)$ & & $\begin{array}{c}19,701 \\
5.23\end{array}$ & 17,54 & 2,14 & $\mathrm{~b}$ & & $0,19 \quad$ a & 0,60 & $0,08 \mathrm{~b}$ & & 07,301 bc & 93,48 & 24,03 & bcde \\
\hline & & & $6.8(n=5)$ & & & 15,80 & 2,56 & b & & & 0,54 & $0,09 \mathrm{~b}$ & & & 89,68 & 21,13 & bcde \\
\hline
\end{tabular}

\section{-A Nivel de Especie}

-Crecimiento Radial: No se evidenció diferencias significativas entre los niveles de pH dentro de los medios BAF y PDA, sólo en el medio MMN donde el pH 6,8 fue diferente a los demás. Tampoco se detectó diferencia entre los medios de cultivo (cuadro 10).

- Velocidad Media de Crecimiento: Presenta un comportamiento similar a la variable CR, no existen diferencias significativas entre los niveles de $\mathrm{pH}$ que se encontraban interactuando dentro de los medios BAF y PDA, mostrando sólo diferencias dentro del medio MMN, con el promedio obtenido a 6.8. En relación al medio $\mathrm{MMN}$ a pH 6,8. este presentó diferencias significativas con los promedios obtenidos para $\mathrm{pH} 4,8$ y 5,3 de este mismo medio, como también, con los medios PDA a pH 5,3 y BAF a pH 6,3 y 6,8 . Respecto al factor $\mathrm{pH}$, la mayor velocidad de crecimiento fue a 5,8 y la menor a 5,3 , ambas estadísticamente diferentes. No se obtuvieron diferencias significativas entre los 3 medios de crecimiento (cuadro 10).

-Biomasa: La mayor producción de biomasa seca se obtuvo en el medio BAF con pH 5,3 y 4,8, pero sin diferencias significativas respecto a BAF con pH 5,8 y 6,3, ni con PDA a pH 5,8. No se detectó diferencias significativas asociadas a los niveles de $\mathrm{pH}$ de los medios PDA y MMN, además de observar un bajo desempeño en peso seco para este último medio de cultivo.

Respecto al factor $\mathrm{pH}$, el ajuste a 5,8 fue el que produjo el mayor valor en peso seco, pero sin diferencias significativas con los ajustes a 5,3 y 4,8. En cuanto al factor medio de cultivo, BAF genera significativamente más biomasa seca que los dos medios restantes (cuadro 10).

-Correlaciones entre Variables: La producción de biomasa presenta una débil correlación con el crecimiento radial $(0,14)$ y con la velocidad media de crecimiento $(0,11)$. 
Cuadro 10. Efecto del medio de cultivo y pH sobre los valores medios (m) y desviaciones estándar (DE) de las variables crecimiento radial, velocidad media de crecimiento y producción de biomasa para Suillus bellinii.

\begin{tabular}{|c|c|c|c|c|c|c|c|c|c|c|c|c|c|c|c|c|c|c|}
\hline \multicolumn{2}{|c|}{ Factores } & \multirow{2}{*}{$\begin{array}{l}\text { Interacción } \\
\text { Medio*Ph }\end{array}$} & \multicolumn{5}{|c|}{ Crecimiento radial (mm) } & \multicolumn{6}{|c|}{ Velocidad media de crecimiento (mm/día) } & \multicolumn{5}{|c|}{ Biomasa (mg) } \\
\hline Medio & \multirow{4}{*}{$\begin{array}{c}\mathrm{pH} \\
\begin{array}{c}4.8 \\
(n=30)\end{array}\end{array}$} & & $\begin{array}{c}\text { Medio } \\
m \pm D E\end{array}$ & $\begin{array}{c}\mathrm{pH} \\
\mathrm{m} \pm \mathrm{DE}\end{array}$ & & $\begin{array}{l}\text { edio }^{*} \mathrm{P} \\
\mathrm{m} \pm \mathrm{DE}\end{array}$ & & $\begin{array}{r}\text { Med } \\
\mathrm{m} \pm \mathrm{D} \\
\end{array}$ & & $\begin{array}{c}\mathrm{pH} \\
\mathrm{m} \pm \mathrm{DE}\end{array}$ & & $\begin{array}{l}\text { ledio* } \mathrm{P} \\
\mathrm{m} \pm \mathrm{DE}\end{array}$ & & $\begin{array}{c}\text { Medio } \\
\mathrm{m} \pm \mathrm{DE}\end{array}$ & $\begin{array}{c}\mathrm{pH} \\
\mathrm{m} \pm \mathrm{DE}\end{array}$ & & $\begin{array}{l}\text { ledio*p } \\
m \pm D E\end{array}$ & \\
\hline \multirow{5}{*}{$\begin{array}{c}\text { PDA } \\
(n=50)\end{array}$} & & $4.8(n=10)$ & \multirow{5}{*}{$\begin{array}{c}18,82 \pm \\
2,57\end{array}$} & \multirow{3}{*}{$\underset{2,74}{18,52 \pm}$} & 18,47 & 2,29 & $a b c$ & \multirow{5}{*}{$\begin{array}{c}0,65 \pm \\
0,09\end{array}$} & \multirow{5}{*}{ a } & \multirow{3}{*}{$\begin{array}{c}0,63 \pm \\
0,09\end{array}$} & 0,64 & 0,08 & $a b c$ & \multirow{5}{*}{${ }_{20,92}^{93,8 \pm} b$} & \multirow{3}{*}{$\underset{37,31}{96,12 \pm} a b c$} & 94,81 & 18,68 & bcde \\
\hline & & $5.3(n=10)$ & & & 18,19 & 3,13 & $a b c$ & & & & 0,62 & 0,10 & bc & & & 100,62 & 20,70 & bcde \\
\hline & & $5.8(n=10)$ & & & 20,86 & 2,59 & $a b$ & & & & 0,73 & 0,09 & $a b$ & & & 105,30 & 24,42 & abcd \\
\hline & \multirow{3}{*}{$\begin{array}{c}5.3 \\
(n=30)\end{array}$} & $6.3(n=10)$ & & \multirow{3}{*}{$\begin{array}{c}18,32 \pm \\
3,63\end{array}$} & 18,70 & 1,68 & $a b c$ & & & \multirow{3}{*}{$\begin{array}{c}0,62 \pm \\
0,12\end{array}$} & 0,65 & 0,06 & $a b c$ & & & 84,52 & 11,41 & cdef \\
\hline & & $6.8(n=10)$ & & & 17,87 & 2,30 & bc & & & & 0,62 & 0,07 & $a b c$ & & 37,10 ab & 83,76 & 21,69 & cdef \\
\hline \multirow{5}{*}{$\begin{array}{l}\text { MMN } \\
(n=50)\end{array}$} & & $4.8(n=10)$ & \multirow{5}{*}{$\begin{array}{c}19,49 \pm \\
4,50\end{array}$} & & 17,26 & 2,60 & $\mathrm{bc}$ & \multirow{5}{*}{\multicolumn{2}{|c|}{$\begin{array}{c}0,66 \pm \\
0,16\end{array}$}} & & 0,59 & 0,09 & $\mathrm{bc}$ & \multirow{5}{*}{${ }_{17,08}^{65,16 \pm} c$} & & 61,12 & 14,44 & $f$ \\
\hline & \multirow{3}{*}{$\begin{array}{c}5.8 \\
(n=30)\end{array}$} & $5.3(n=10)$ & & & 17,49 & 5,36 & bc & & & \multirow{3}{*}{$\begin{array}{l}0,7 \pm \\
0,12\end{array}$} & 0,58 & 0,18 & $\mathrm{bc}$ & & & 56,33 & 18,18 & $f$ \\
\hline & & $5.8(n=10)$ & & $\underset{3,56}{20,4 / \pm}$ a & 20,59 & 4,81 & $a b$ & & & & 0,70 & 0,17 & $a b c$ & & $\begin{array}{c}100,84 \pm \\
33,44\end{array}$ & 71,56 & 20,41 & ef \\
\hline & & $6.3(n=10)$ & & & 19,35 & 2,84 & $a b c$ & & & & 0,66 & 0,11 & $a b c$ & & & 62,25 & 12,11 & $f$ \\
\hline & \multirow{3}{*}{$\begin{array}{c}6.3 \\
(n=30)\end{array}$} & $6.8(n=10)$ & & \multirow{3}{*}{$\underset{2,19}{18,47 \pm}$ a } & 22,74 & 4,56 & a & & & \multirow{3}{*}{$\begin{array}{c}0,63 \pm \\
0,08\end{array}$} & 0,78 & 0,16 & a & & \multirow{3}{*}{$\underset{24,46}{-84,43 \pm}$ bc } & 74,56 & 15,12 & def \\
\hline \multirow{5}{*}{$\begin{array}{c}\text { BAF } \\
(n=50)\end{array}$} & & $4.8(n=10)$ & \multirow{5}{*}{$\begin{array}{c}18,46 \pm \\
2,79\end{array}$} & & 19,82 & 2,91 & $a b c$ & & & & 0,67 & 0,09 & $a b c$ & & & 132,42 & 33,18 & a \\
\hline & & $5.3(n=10)$ & & & 19,29 & 1,48 & $a b c$ & & & & 0,65 & 0,05 & $a b c$ & & & 134,18 & 16,94 & $a$ \\
\hline & & $5.8(n=10)$ & & & 19,97 & 3,24 & $a b c$ & 0,09 & $a$ & & 0,67 & 0,11 & $a b c$ & ${ }_{30,54}^{117,03 \pm}$ a & & 125,67 & 30,51 & $a b$ \\
\hline & $(n=30)$ & $6.3(n=10)$ & & $\underset{4,26}{10,011}$ a & 17,37 & 1,52 & bc & & & 0,15 & 0,59 & 0,06 & $\mathrm{bc}$ & & ${ }_{18,79} \mathrm{C}$ & 106.53 & 23,71 & $a b c$ \\
\hline & & $6.8(n=10)$ & & & 15,83 & 2,09 & $\mathrm{c}$ & & & & 0,54 & 0,08 & $\mathrm{c}$ & & & 86,33 & 18,80 & cdef \\
\hline
\end{tabular}

\section{DISCUSIÓN}

El efecto del medio de cultivo y del pH sobre la respuesta en crecimiento y producción de biomasa de las cepas y especies de hongos evaluadas indican variaciones en las estrategias mediante la cual estos organismos enfrentan los cambios en su medio de crecimiento. En algunos casos muestran respuestas distintas entre cepas y entre especies, influenciados por las interacciones que se presentan entre los factores medioambientales y la variabilidad genética que presenta cada especie. Por lo mismo, los resultados se deben considerar en forma particular para cada cepa evaluada.

Se han realizado estudios para evaluar parámetros medioambientales con diferentes cepas de especies micorrícicas, con resultados muy diversos debido principalmente a la variabilidad genética que presentan dichos microrganismos y a las condiciones de sitio donde se desarrollan. Esto es reafirmado por los investigadores Islam \& Ohga (2013), quienes indican que para llegar a producir inoculantes fúngicos a gran escala, es necesario definir la composición óptima del medio de cultivo para cada hongo tomando en cuenta las diferentes cepas sobre una gran variación de condiciones medioambientales.

Con respecto al crecimiento radial de $S$. granulatus en diferentes medios de cultivo y niveles de $\mathrm{pH}$, Murrieta-Hernández et al. (2014) señalan que PDA a pH 5,8 fue el mejor tratamiento, coincidiendo con lo observado en este estudio para las dos cepas evaluadas. Los autores también obtienen un bajo crecimiento en el medio MMN en relación a BAF y PDA, aspecto que también coincide con lo observado en el presente estudio y en las investigaciones efectuadas por Torres \& Honrubia (1991).

Gonzalez et al. (2015) trabajando con diferentes medios de cultivos y valores de $\mathrm{pH}$ en Suillus luteus, mencionan al medio BAF como uno de los que promueve el mayor crecimiento a pH de 4,8 y 5,8, lo que concuerda parcialmente con los resultados de este estudio. En efecto, la cepa IF732002 obtuvo los mayores crecimientos en este medio con pH 5,8, mientras que para la cepa IF1618002 fue a pH 6,3.

Sianard et al. (2010) trabajando con diferentes ajustes de $\mathrm{pH}$ en medio BAF, concluyen que para $S$. bellinii el mayor crecimiento se produjo a $\mathrm{pH} 4,6$ y que para $S$. granulatus fue a 5,6 . Estos antecedentes concuerdan en gran medida con lo obtenido en una de las cepas de $S$. bellinii (IF633001), y con lo observado en ambas cepas de $S$. granulatus, que lograron el mayor crecimiento en un $\mathrm{pH}$ muy similar $(5,8)$. En cambio, Murrieta-Hernández et al. (2014) señala que los dos valores de pH que evaluaron en $S$. granulatus $(4,8$ y 5,8), no indujeron diferencias de crecimiento, lo que difiere con lo obtenido en ese ensayo. Además, estos investigadores, mencionan que el medio PDA produjo el mayor crecimiento miceliar, lo que es similar a lo observado con la cepa IF937002, aunque esta presentó también un rendimiento parecido en medio BAF; en tanto que para la cepa IF1616002 fue sólo en el medio BAF. Otros estudios realizados por Santelices et al. (2012) utilizando valores de $\mathrm{pH}$ de 5,0, 5,8 y 6,5 en los medio MMN y MEAa2\% (agar con extracto de Malta al $2 \%$ ), hacen también referencia a que no obtuvieron diferencias de crecimiento miceliar que sean significativas a nivel de $\mathrm{pH}$ y medio de cultivo 
para S. luteus, lo que es bastante coincidente con lo obtenido en medio MMN para esta especie. GarcíaRodriguez et al. (2017), estudiando los efectos del pH a 4,8,5,3, 5,8, 6,3 y 6,8 sobre el crecimiento radial de cepas de $S$. luteus y $S$. granulatus en medio MMN, mencionan comportamientos muy parecidos entre ellas, lo que coincide con lo observado en este estudio a excepción de la cepa de S. luteus IF 732002 que presenta diferencias significativas entre el medio MMN a 6,8 y MMN a 4,8. Trabajos realizados por Lazarevic et al. (2016) en relación a los efectos del pH sobre el crecimiento miceliar de $S$. granulatus, utilizando el medio nutritivo $\mathrm{MMN}$, determinaron que el mayor crecimiento se obtenía con rangos de $\mathrm{pH}$ entre 4,0 y 5,2, lo que no concuerda con lo observado en este estudio, cuyos resultados señalan mejores crecimientos para el medio MMN con pH de 6,8 y 5,8, para las cepas IF937002 y IF1616002, respectivamente. Las dificultades de encontrar un patrón de comportamiento fueron mencionadas por Garcia-Rodriguez et al. (2017) al analizar la variable CR en las especies S. granulatus y S. luteus. Además, factores como el $\mathrm{pH}$, pueden determinar biológicamente el tipo de organismo capaz de desarrollarse en un sustrato, debido a su influencia significativa en la disponibilidad de nutrientes (Pereira et al., 2007).

En lo relativo a la velocidad media de crecimiento en medio BAF, con pH ajustado a 4,6, 5,6, 6,6 y 7,6, Sianard et al. (2010) indican que $S$. bellinii presentó su máxima velocidad a $\mathrm{pH} 4,6$, y $S$. granulatus a pH 6,6. Estos resultados son parcialmente coincidentes con los obtenidos en este estudio en medio BAF para las cepas de $S$. bellinii IF633001 y IF713001, las que lograron la mayor velocidad de crecimiento en BAF con pH 4,8 y 5,8 , respectivamente, mientras que para $S$. granulatus el máximo valor obtenido para las cepas IF16116002 y IF937002 fue con pH 5,8.

Murrieta-Hernández, et al. (2014) sugieren el medio BAF como medio de producción masiva de micelio para $S$. granulatus, debido a la mayor velocidad de crecimiento que se consigue en dicho medio. Pereira et al. (2007) también obtienen la mayor velocidad en BAF con pH 4,8, y esta decrece a medida que disminuye la acidez del medio, resultado distinto a lo obtenido para las cepas evaluadas en el presente estudio, las que obtuvieron su mayor valor entre $\mathrm{pH}$ 5,8 y 6,3 en el mismo medio BAF, y valores aún mayores para $\mathrm{pH} 5,8$ en medio PDA y 6,8 en MMN para las cepas IF1618002 y IF732002, respectivamente.

En relación a la producción de biomasa seca, diversos investigadores coinciden en que los medios PDA y BAF resultaron ser significativamente más efectivos para la especie S. luteus (Fernández-Miranda, 2008; Murrieta-Hernández, et al., 2014; Gonzalez et al., 2015); por el contrario, mencionan al medio MMN como el que produce los menores rendimientos en biomasa. Sobre este particular Molina y Palmer (1982), afirman que el medio de cultivo más adecuado para un hongo ectomicorrícico, es el que le aporte los requerimientos nutritivos que obtienen de su hospedante al establecer la simbiosis, lo cual marcaría el éxito o fracaso en el cultivo de estas especies bajo condiciones controladas. Bajo esta premisa, en el contexto de los resultados obtenidos y buscando una metodología que permita generar la mayor cantidad de biomasa, se puede indicar al medio BAF como el mejor medio para su utilización en la producción miceliar, debido a su buen desempeño en generar la mayor cantidad de materia seca, para las seis cepas evaluadas. Se agrega para este uso el medio PDA, de menor costo, y que en 4 de las seis cepas evaluadas mostro rendimientos similares y sin diferencias significativas con el medio BAF.

La mayor producción de biomasa seca para Suillus luteus se obtuvo para pH igual o mayor a 5,3, con $154,54 \mathrm{mg}$ a pH 5,8 en medio BAF. En S. granulatus su mejor producción se obtuvo entre PH 5,3 y 6,3, con 173,84 mg a pH 5,8 en medio BAF. En S. bellinii la mayor producción de biomasa seca se logró a pH entre 4,8 y 5,8 , aunque también se alcanzó buen rendimiento a $\mathrm{pH} 6,3$, como es el caso de la cepa IF 71300; en esta última especie su valor máximo alcanzó 149,50 mg a pH 4,8 en medio BAF.

García-Rodriguez et al. (2017) estudiaron el efecto de diferentes valores de pH sobre la producción de biomasa, determinando que no existían diferencias significativas entre los tratamientos para cepas de $S$. luteus y S. granulatus; esto coincide con lo obtenido en este estudio con la cepa IF1618002 de S. Iuteus, sin embargo, los resultados obtenidos también muestran un efecto del pH en la producción de materia seca dependiendo de la cepa evaluada y del medio que se utilice para la evaluación. Es así como la cepa IF732002 de S. luteus, exhibió un crecimiento menor a pH 4,8 y significativamente diferente a los otros niveles de $\mathrm{pH}$ dentro del medio BAF, no así en los medios MMN y PDA. En las dos cepas de $S$. granulatus, se observó una menor producción de biomasa a pH 4,8 en todos los medios utilizados y obteniendo los mayores montos a pH 5,8 en medio BAF. Por otro lado, existe otros rangos de $\mathrm{pH}$ en los 
cuales se ha obtenido un aumento de biomasa, como lo han observado Sánchez et al. (2001) para $S$. granulatus y $S$. luteus a un $\mathrm{pH}$ de 8,5. De acuerdo a lo observado en este estudio y lo obtenido por Hung \& Trappe (1983), los rangos de $\mathrm{pH}$ que generan un buen crecimiento varían drásticamente entre especies como entre cepas dentro de las especies.

Respecto a las relaciones entre las variables de crecimiento (CR, VCM) y la biomasa seca (B), se obtuvo resultados muy variados. Una alta correlación fue observada para las cepas de Suillus granulatus entre la variable $\mathrm{CR}$ y $\mathrm{B}$, pero no así para $S$. luteus, que presentó una baja correlación para sus dos cepas estudiadas. En Suillus bellinii esta correlación fue moderada para la cepa IF713001 y nula para la cepa IF633001. Se concluye que el crecimiento radial no es buen indicador de la producción de biomasa seca para las especies S. luteus y S. bellinii, cuyas correlaciones son bajas a nulas entre la variable CR y B. En $S$. granulatus, a pesar de obtener una alta correlación no se puede asegurar este mismo comportamiento para todas las cepas de esta especie. Un indicador confiable del comportamiento de una determinada cepa sería la producción de biomasa, la cual no necesariamente puede estimarse a partir del crecimiento radial. Esto también ha sido mencionado por Santiago-Martínez et al. (1995), los cuales señalan que los mayores crecimientos en diámetro de la cepa bajo un cultivo in vitro, no siempre corresponden con la mayor producción de biomasa, aspecto que es de importancia debido al frecuente empleo de este parámetro como único elemento para evaluar el crecimiento de la cepa.

\section{CONCLUSIONES}

El desarrollo de los hongos micorrícicos depende de diversos factores medio ambientales. Si se analiza a nivel de especie, y más aun a nivel de cepa o ecotipo, estos poseen sus propias limitaciones frente a determinadas condiciones ambientales (Sianard, et al., 2010). Por lo tanto, el estudio de factores ambientales como el nivel de $\mathrm{pH}$ y los medios de cultivos para la masificación de hongos y/o cepas, son necesarios para poder conocerlos, pues sus comportamientos difieren al interactuar con cada uno de estos parámetros (Honrubia et al., 1992; Pereira, 2002; Vázquez- García et al., 2002). Conocer las relaciones entre tales parámetros y las respuestas de cada hongo o cepa en particular, permitirá perfeccionar la producción de micelios de hongos ectomicorrícicos.

Si bien bajo ciertas condiciones de cultivo algunas cepas presentaron mejor rendimiento que otras en términos de crecimiento radial, velocidad de crecimiento y producción de biomasa, su selección final para ser utilizadas en actividades de micorrización de plantas dependerá de su desempeño en vivero y su comportamiento en campo. Hung y Trappe (1983), citando a varios investigadores, afirman que el efecto del $\mathrm{pH}$ sobre el crecimiento fúngico in vitro, debe ser interpretado con precaución, debido a que el desempeño del hongo puede ser afectado por múltiples factores que pueden hacer variar los resultados, como la duración del experimento, las fuentes de nitrógeno, la inclusión de sales de hierro antes o después del autoclavado del medio, y otros. Estos mismos autores, señalan que, en igualdad de condiciones, un aislado que crezca razonablemente bien en un amplio rango de valores de $\mathrm{pH}$ sería preferible para trabajos de masificación e inoculación en vivero a uno que crezca bien solo en un rango restringido.

De acuerdo a comportamiento observando en las cepas y especies evaluadas, se plantea la necesidad de recolectar y testear gran cantidad de material fúngico, que recoja la máxima variabilidad bajo diversos ambientes, con el fin de seleccionar cepas específicas para sitios específicos, ajustando en forma paralela los protocolos que permitan desarrollar una producción operativa de inoculantes a gran escala para satisfacer necesidades de micorrización de viveros. Esta necesidad de selección se debe a la variación genética de las especies fúngicas, la que debe ser considerada al momento de seleccionar especies y cepas, para la elaboración de inoculantes de plantas de vivero (Pera et al., 1998).

$\mathrm{El} \mathrm{pH}$ juega un papel importante en el crecimiento de hongos ectomicorrícicos, aspecto que fue corroborado en este trabajo, donde se observó cepas más acidófilas que otras. En otros casos este factor no presenta efecto significativo dentro de cada medio de cultivo, pero si en la interacción con el mismo, lo que podría deberse a la influencia del $\mathrm{pH}$ sobre la disponibilidad de algunos nutrientes en el medio utilizado. En efecto, Pereira et al. (2007) mencionan que el pH tiene una influencia significativa en la disponibilidad de nutrientes, pudiendo determinar biológicamente el tipo de organismo capaz de desarrollarse en un suelo o sustrato. Por su parte, Willenborg et al. (1990), mencionó que los hongos 
ectomicorrícicos tienen, en general, una naturaleza acidófila cuando se cultivan en condiciones de cultivo puro.

Los resultados obtenidos sugieren que los medios BAF y PDA, y valores de pH cercanos a 5,8, serían combinaciones adecuadas para la masificación miceliar orientada a la producción de inoculantes para viveros forestales. Sin embargo, no se debe descartar el factor de la variación genética, que podría hacer necesario el uso de uno u otro medio de cultivo y distintos niveles de $\mathrm{pH}$, para cada una de las especies y cepas a masificar.

\section{REFERENCIAS}

Blanco, D., Fajardo, J., Verde, A. \& Rodríguez, C. (2012). Etnomicología de los hongos del género Suillus, una visión global. Bol. Soc. Micol. Madrid, 36. Pp: 175 - 186.

Brundrett, M. \& Cairney, J. (2002). Ectomycorrhizas in plant communities. En: Microorganisms in plant conservation and biodiversity. Springer, Dordrecht. Pp: 105-150. https://doi.org/10.1007/0-306-48099-9_5

Bruns, T.D., Tan, J., Bidartando, M., Szaro, T.M. \& Redecker, D. (2002). Sunvival of Suillus pungens and Amanita francheti ectomyconhizal genets was rare or absent after a stand-replacing fire. New Phytologist, 155. Pp: 517-523. https://doi.org/10.1046/j.14698137.2002.00468.x

Chung, P. (2020). Captura, aislación y evaluación del crecimiento de material fúngico de la región de Nuble para su incorporación al Banco de Hongos Comestibles del Instituto Forestal. Ciencia e Investigación Forestal INFOR Chile. Volumen $26 \mathrm{~N}^{\circ} 3$ Diciembre 2020 pp: 65-92 https://doi.org/10.52904/0718-4646.2020.538

Fernández-Miranda, E., Alvarado, P., Alonso-Graña, M., Majada, J. \& Casares, A. (2008). Influencia de las condiciones culturales en el crecimiento de diferentes hongos ectomicorrícicos. Actas de la IV Reunión sobre Repoblaciones Forestales. Cuad. Soc. Esp. Cienc. For., 28. Pp: 207-211.

García-Rodriguez, J., Pérez-Moreno, J., Ríos-Leal, D., Sáez-Delgado, P., Atala-Bianchi, C., Sánchez-Olate, M. \& Pereira-Cancino, G. (2017). Crecimiento in vitro de hongos ectomicorrícicos asociados con plantaciones de Pinus radiata en Chile. Revista Fitotec. Mexico., 40(4): 415-423. https://doi.org/10.35196/rfm.2017.4.415-423

García-Rodríguez J., Pérez-Moreno, J., Aldrete, A., Cetina-Alcalá, V. \& Vaquera-Huerta, H. (2006). Caracterización del hongo silvestre ectomicorrícico Pisolithus tinctorius (Pers.) Coke et Couch en cultivo y en simbiosis con eucalipto y pino. Agrociencia 40:665-676.

González, M., Quiroz, I., Travieso, R., Chung, P. \& García, E. (2015). Determinación de medios de cultivo y pH para la masificación in vitro de cepas de Suillus luteus Aubl. asociadas a Pinus radiata D. Don y Scleroderma citrinum Pers. asociadas a Eucalyptus globulus Labill. de la región del Biobío, Chile. Revista Árvore, 39. Pp: 105-113. https://doi.org/10.1590/0100-67622015000100010

Honrubia, M., Torres, P., Díaz, G. \& Cano, A. (1992). Manual para micorrizar plantas en viveros forestales. Madrid. Ministerio de Agricultura, Pesca y Alimentación

Hung, L. \& Trappe, J. (1983). Growth variation between and within species of ectomycorrhizal fungi in response to $\mathrm{pH}$ in vitro. Mycologia 75:234-241. https://doi.org/10.1080/00275514.1983.12021660

INFOR. (2020). Estadísticas forestales. En: https://wef.infor.cl/. Consulta: 10 marzo, 2021

Islam, F. \& Ohga, S. (2013). Effects of media formulation on the growth and morphology of ectomycorrhizae and their association with host plant. ISRN Agronomy. Vol. 2013, Article ID 317903, 12 p. Hindawi Publishing Corporation. https://doi.org/10.1155/2013/317903

Lazarevic J., Stojičić, D. \& Keča, N. (2016). Effects of temperature, pH, carbon and nitrogen sources on growth of in vitro cultures of ectomycorrhizal isolates from Pinus heldreichii forest. Forest Systems, 25. Pp: 1-10. https://doi.org/10.5424/fs/2016251-07036

Marx, D.H. (1969). The influence of ectotrophic fungi on the resistance of pine roots to pathogenic infections. I. Antagonism of mycorrhizal fungi to root pathogenic fungi and soil bacteria. Phytopathology, v.59, p.153-163.

Molina, R. \& Palmer, J. (1982). Isolation, maintenance and pure culture manipulation of ectomycorrhizal fungi. En: Methods and Principles of Mycorrhizal Research. N.C. Schenck (Ed) American Phytopathological Soc. St. Paul.

Montgomery, D. (1984). Design and Analysis of Experiments. John Wiley \& Sons. New York. 649 p.

Moser, M. (1960). Die Gattung Phlegmacium. Die Pilze Mitteleupora's 4. J. Bad Heilbrunn. 
Murrieta-Hernández, D.M., Noa-Carrazana, J.C., Mata-Rosas, M., Pineda-López, M del R., Zulueta-Rodríguez, R. \& Flores-Estévez, N. (2014). Efecto del medio de cultivo en el desarrollo de Suillus granulatus (L.) Roussel y $S$. brevipes (Pk.) Kuntze. Revista Chapingo Serie Ciencias Forestales y del Ambiente, 20. Pp: 101-107. https://doi.org/10.5154/r.rchscfa.2013.06.021

Pera, J., Álvarez, L. \& Parlade, J. (1998). Eficacia del inóculo miceliar de 17 especies de hongos ectomicorrícicos para la micorrización controlada de: Pinus pinaster, Pinus radiata y Pseudotsuga menziesii, en contenedor. Invest. Agr.: Sist. Rec. For., 7(1 y 2): 139-153.

Pereira, G., Herrera, J., Machuca, A. \& Sánchez, M. (2007). Efecto del pH sobre el crecimiento in vitro de hongos ectomicorrícicos recolectados de plantaciones de Pinus radiata. Bosque 28. Pp: $215-219$. https://doi.org/10.4067/S0717-92002007000300005

Pereira, G. (2002). Micorrizas, suelos degradados y silvicultura de precisión. Chile Forestal. 276. Pp: 27- 29.

Sánchez, F., Honrubia, M. \& Torres, P. (2001) Effects of $\mathrm{pH}$, water stress and temperature on in vitro cultures of ectomycorrhizal fungi from Mediterranean forests. Cryptogamie Mycologie, 22. Pp: $243-258$. https://doi.org/10.1016/S0181-1584(01)01076-4

Santelices, R., Espinoza, S., Brunel, N. \& Palfner, G. (2012). Effect of the geographical origin, culture media, and pH on the growth dynamic of the edible ectomycohrrizal mushroom Suillus luteus. Cien. Inv. Agr., 39(2): $369-376$. https://doi.org/10.4067/S0718-16202012000200013

Santiago-Martínez, G., Varela, L., Estrada-Torres, A. \& Cuaxilo, V. (1995). Efecto de seis medios de cultivo sobre el crecimiento de tres cepas de Pisolithus tinctorius. Revista Mexicana de Micología, 11. Pp: 57-68. https://dx.doi.org/10.33885/sf.1995.3.829

Santiago-Martínez G., Estrada-Torres, A., Varela, L. \& Herrera, T. (2003). Crecimiento en siete medios nutritivos y síntesis in vitro de una cepa de Laccaria bicolor. Agrociencia, 37(6): 575-584.

Sianard, F., Pangou, S. \& Mountanda, A. (2010). Influencia del pH el en desarrollo in vitro de cinco especies de hongos ectomicorrícicos. Centro Agrícola, 37(1): 23-28.

Torres, P. \& Honrubia, M. (1991). Dinámica de crecimiento y caracterización de algunos hongos ectomicorrícicos en cultivo. Cryptogamie Mycologie, 12. Pp: 183-192.

Vázquez-García, A., Santiago-Martínez, G \& Estrada-Torres, A. (2002). Influencia del pH en el crecimiento de quince cepas de hongos ectomicorrizógenos. Anales del Instituto de Biología. Serie Botánica, 73. Pp: 1-15.

Willenborg A., Schmitz, D. \& Lelley, J. (1990) Effects of environmental stress factors on ectomycorrhizal fungi in vitro. Canadian Journal of Botany, 68. Pp: 1741-1746. http://doi.org/10.1139/b90-224 